\title{
Sesamol Attenuates Neuroinflammation by Regulating the AMPK/SIRT1/NF- $\kappa$ B Signaling Pathway after Spinal Cord Injury in Mice
}

\author{
Xiaochu Feng, Xianghang Chen, Muhammad Zaeem, Wanying Zhang, Liwan Song, \\ Lulu Chen, Joana Mubwandarikwa, Xiangxiang Chen, Jian Xiao $\mathbb{D}$, Ling Xie $\mathbb{D}$, \\ and Keyong Ye
}

Department of Orthopaedics, Affiliated Pingyang Hospital and School of Pharmaceutical Science, Wenzhou Medical University, Wenzhou, Zhejiang 325000, China

Correspondence should be addressed to Jian Xiao; xfxj2000@126.com, Ling Xie; xieling0612@163.com, and Keyong Ye; yeky12600@126.com

Received 21 August 2021; Accepted 23 November 2021; Published 6 January 2022

Academic Editor: Zonghao Tang

Copyright (C) 2022 Xiaochu Feng et al. This is an open access article distributed under the Creative Commons Attribution License, which permits unrestricted use, distribution, and reproduction in any medium, provided the original work is properly cited.

\begin{abstract}
Inflammation is one of the crucial mechanisms mediating spinal cord injury (SCI) progress. Sesamol, a component of sesame oil, has antiinflammatory activity, but its mechanism in SCI remains unclear. We investigated if the AMPK/SIRT1/NF- $\kappa$ B pathway participated in anti-inflammation of sesamol in SCI. Sesamol could inhibit neuronal apoptosis, reduce neuroinflammation, enhance M2 phenotype microglial polarization, and improved motor function recovery in mice after SCI. Furthermore, sesamol increased SIRT1 protein expression and p-AMPK/AMPK ratio, while it downregulated the p-p65/p65 ratio, indicating that sesamol treatment upregulated the AMPK/SIRT1 pathway and inhibited NF- $\kappa$ B activation. However, these effects were blocked by compound $\mathrm{C}$ which is a specific AMPK inhibitor. Together, the study suggests that sesamol is a potential drug for antineuroinflammation and improving locomotor functional recovery through regulation of the AMPK/SIRT1/NF- $\kappa$ B pathway in SCI.
\end{abstract}

\section{Introduction}

Spinal cord injury (SCI) is severe central nervous system (CNS) damage, and more than 250000 patients suffer from it every year [1]. However, there are few effective treatments for SCI. As a grievous neurological disease, SCI seriously destroys the function of motor and sensory neurons; paralysis caused by SCI brings huge medical and economic burden to the patients and their families $[2,3]$.

Traumatic SCI involves primary mechanical insult and the secondary injury [4]. The primary insult makes a direct crash to the spinal cord and damages cells, which also leads to a series of complex secondary injury molecular events, including toxic oxidative stress, excessive microglial activation, continuous inflammation, and rampant apoptosis [2, 5]. Neuroinflammation is one of the dominating mechanisms mediating secondary SCI progress. Microglia, the
CNS-resident immune cells, maintain a resting state under control condition and become activated in response to local CNS injury [6]. There are two phenotypes of microglia called M1 and M2 after activation [7, 8]. M1 microglia are detrimental and can generate a lot of proinflammatory cytokines, including TNF- $\alpha$ and IL- 6 , while the M2 phenotype is protective and upregulates anti-inflammatory cytokines, including IL-10, which contribute to tissue regeneration and wound healing [9]. The resident microglia are activated after SCI, and then, a lot of inflammatory cytokines produced in the early stage of SCI, which will cause neuronal apoptosis, aggravate the injury and make recovery more difficult [10-12]. Therefore, it is especially important to alleviate inflammatory response in the early stage of injury.

Sesamol, a component of sesame oil [13], has been proven to have effects of anti-inflammation and neuroprotection [13-15]. As a member of the sirtuin (SIRTs) family, 
SIRT1 has been proven to have positive impacts on antiaging, anti-inflammation, and reducing oxidative stress damage [16-19]. AMP-activated protein kinase (AMPK) is a critical modulator of cellular energy, which coordinates numerous pathways to maintain energy homeostasis [20]. A number of evidences have indicated that AMPK upregulates protein expression of SIRT1 and inhibits inflammation under pathological conditions [16, 21, 22]. SIRT1, as a downstream protein of the AMPK signaling pathway, also plays a part in anti-inflammation via inhibiting $\mathrm{NF}-\kappa \mathrm{B}$ [23-25]. The previous studies have proven that sesamol plays roles in anti-inflammation by suppressing NF- $\kappa \mathrm{B}$ activation and upregulating AMPK signaling $[26,27]$ and attenuating oxidative stress via activation of SIRT1 [28].

Sesamol has potential therapeutic use in SCI, but the underlying mechanism of sesamol in SCI remains poorly understood. This study confirmed the effect and mechanism of sesamol in anti-inflammation in mice following SCI and provided evidence for the potential clinical use of sesamol in SCI.

\section{Materials and Methods}

2.1. Animals. Healthy $8 \sim 10$-week-old male C57BL/6J mice (weighing 20 25 g) were used in this study. All mice lived in standard housing conditions and can drink and eat freely. Animal experimental operations were performed according to protocols authorized by the Laboratory Animal Ethics Committee of Wenzhou Medical University (no. wydw2018-0043).

2.2. SCI and Drug Treatments. The SCI protocol for adult mice under sterile conditions is described previously. Shortly, animals were anesthetized with $1 \%$ pentobarbital sodium (50 mg/kg, i.p.) before surgery. Skin and muscles near spinous processes were incised to expose the dorsal cord in mice. After a laminectomy at the T9-T10 level, a $10 \mathrm{~g}$ weight from $20 \mathrm{~mm}$ height was fallen onto the exposed spinal cord by a modified New York University impactor to induce a moderate SCI [29]. As a control, mice in the sham group did not suffer from SCI after laminectomy and did not receive medication. After SCI surgery, the mouse bladders were manually emptied every morning and evening until bladder function returned to normal.

All mice are randomly assigned, and mice suffering from SCI were randomly arranged to two groups: SCI and SCI+sesamol. Mice in the SCI+sesamol group were administrated daily with sesamol $(10 \mathrm{mg} / \mathrm{kg}$, i.p.) dissolved in saline for 28 days, and animals in the SCI group received the same volume of normal saline every day. Mice were sacrificed, and the damaged spinal cord was taken out for analysis at the corresponding time point after SCI. The time points of administration and experimental arrangement for mice are shown in Figure 1(a).

2.3. Cell Culture and Intervention. BV2 cells were cultivated in DMEM supplemented with $10 \%$ fetal bovine serum and $1 \%$ penicillin and streptomycin mixture in a humidified incubator with $5 \% \mathrm{CO}_{2}$ at $37^{\circ} \mathrm{C}$. BV2 cells were arranged to control, lipopolysaccharide (LPS), LPS+sesamol, and compound C (LPS+sesamol+compound C) groups. Cells were incubated with $10 \mu \mathrm{M}$ sesamol dissolved in PBS for $2 \mathrm{~h}$ and then suffered from LPS $(1 \mu \mathrm{g} / \mathrm{mL})$ for $24 \mathrm{~h}$ to stimulate inflammation. To explore whether the AMPK pathway participates in anti-inflammation of sesamol, cells were pretreated with compound C (a specific AMPK inhibitor) for $2 \mathrm{~h}$ before sesamol treatment.

2.4. Cell Viability Test. BV2 cells were incubated in a 96-well cell culture plate with 10000 cells per well for $24 \mathrm{~h}$ and then administrated with LPS combined with different concentrations of sesamol. The cell viability was detected with Cell Counting Kit-8 (CCK-8) according to the instructions. Shortly, after incubation, cells were further cultured for $2 \mathrm{~h}$ in $90 \mu \mathrm{L}$ of fresh DMEM supplemented with $10 \mu \mathrm{L}$ of CCK-8 solution. At last, optical density (OD) values at $450 \mathrm{~nm}$ were measured using a microplate reader. Six replicate wells of cells were arranged in each group.

2.5. Western Blot. After transcardial perfusion of saline, approximately $1 \mathrm{~cm}$ length of the spinal cord adjacent to the injury center was collected. Tissues and BV2 cells were lysed with a protein extraction reagent containing protease inhibitors and then centrifuged to get proteins. Whole tissue lysate $(60 \mu \mathrm{g})$ or cell lysate $(20 \mu \mathrm{g})$ was separated by $8 \% \sim 12 \%$ SDS-PAGE, and then, proteins were blotted onto the PVDF membrane. Blocked with $5 \%(w / v)$ nonfat milk, the membrane was incubated at $4^{\circ} \mathrm{C}$ overnight using primary antibodies corresponding to these proteins: TNF- $\alpha$, IL-6, Bax, CD86, CD206, p-AMPK (Thr172), AMPK, Bcl-2, SIRT1, p-p65 (S536), p65, and cleaved caspase-3, and further immersed in the corresponding HRP-conjugated secondary antibody for $1.5 \mathrm{~h}$ at room temperature (RT). At last, signal was visualized by using the ChemiDoc ${ }^{\mathrm{TM}} \mathrm{XRS}^{+}$imaging system (Bio-Rad), and the band intensity was analyzed by using Image Lab 5.2 software (Bio-Rad).

2.6. Immunofluorescence. For immunofluorescence, haematoxylin-eosin (HE), and Nissl staining, animals underwent transcardial perfusion with $4 \%(w / v)$ paraformaldehyde (PFA), and then, the injured spinal cord tissue was taken out and fixed with $4 \%$ PFA overnight. BV2 cell climbing slices were also fixed with $4 \%$ PFA for $15 \mathrm{~min}$. The tissues were then dehydrated and immersed in paraffin. Next, tissues were sliced up to $5 \mu \mathrm{m}$ thickness of sections, and then, the slices were dewaxed using xylene and hydrated with 100, 90, 80, and 70\% ethanol. After high-pressure antigen retrieval, tissue slices or BV2 cell climbing slices were blocked with $5 \%(w / v)$ BSA for $30 \mathrm{~min}$ at $37^{\circ} \mathrm{C}$. Next, the slices were treated with primary antibodies at $4^{\circ} \mathrm{C}$ overnight as follows: NeuN, cleaved caspase-3, Iba1, CD206, SIRT1, and p65. Next, the sections or cell climbing slices were further incubated with a proper fluorescenceconjugated secondary antibody at RT for $1 \mathrm{~h}$. Finally, DAPI was used for cellular nuclear staining. The images were visualized by using a Nikon ECLIPSE80i microscope.

2.7. HE Staining and Nissl Staining. Injured spinal cord sections were successively dewaxed and hydrated according to protocols described above. A part of tissue slices was used for $\mathrm{HE}$ staining with an $\mathrm{HE}$ kit, and other slices were stained 


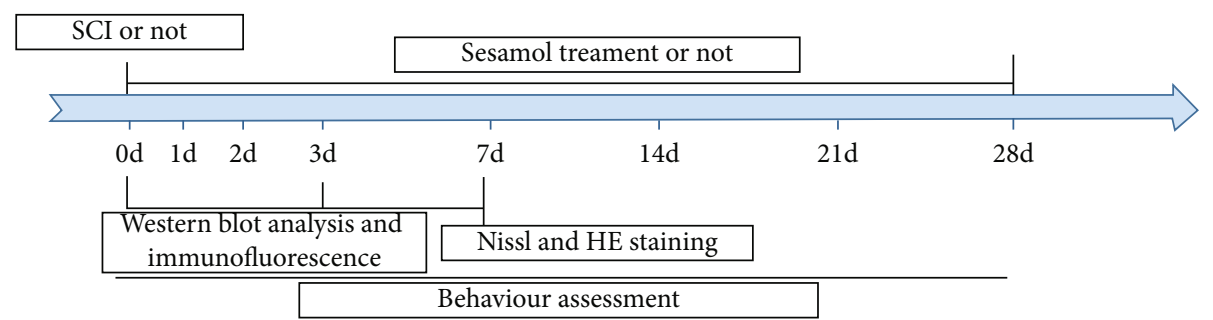

(a)
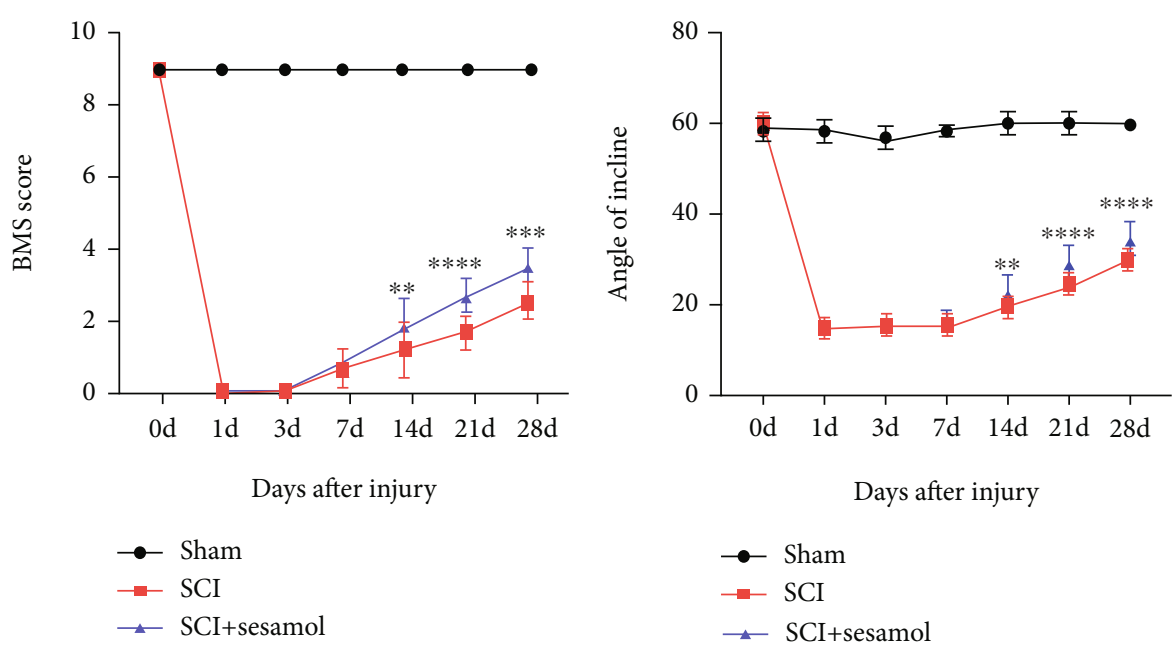

(b)

(c)

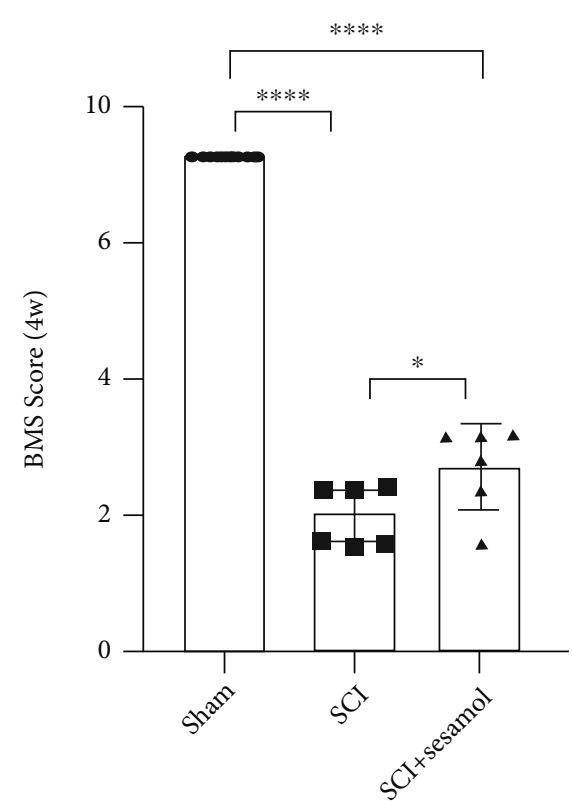

(d)

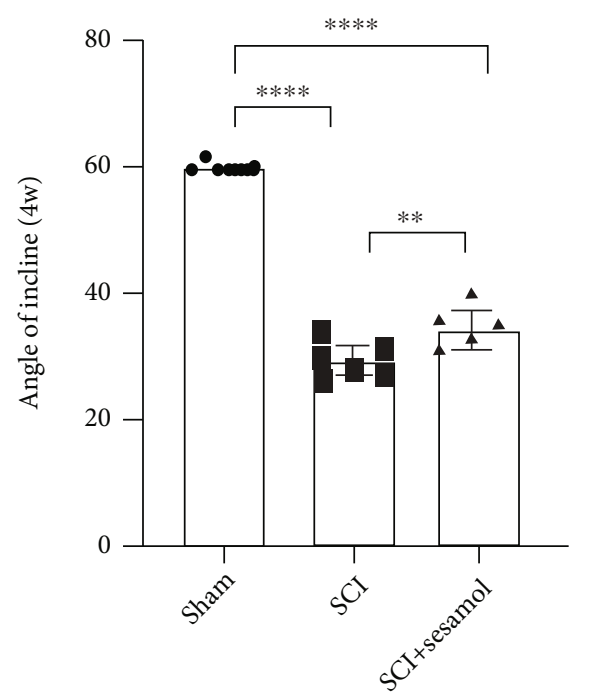

(e)

Figure 1: Continued. 


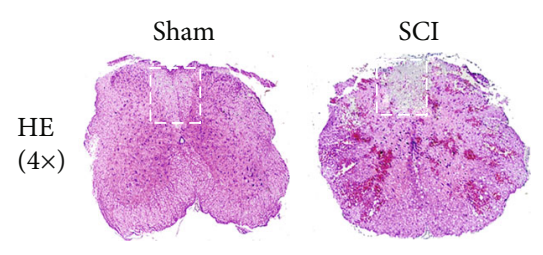

(f)

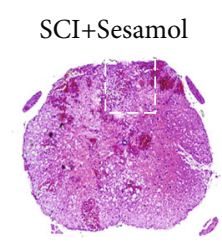

$(20 \times)$

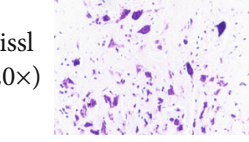

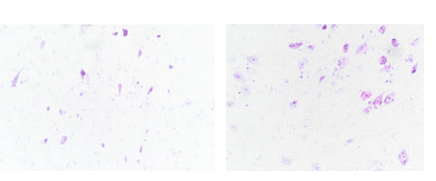

(g)

FIgURE 1: Sesamol evaluates histological outcomes and promotes locomotor functional restoration in mice suffering from SCI. (a) Time schedule of experimental design and analysis in mice. (b) The BMS scores of mice in each group at $0,1,3,7,14,21$, and 28 dpi. (c) Result of the inclined plane test of mice from different group at 28 dpi. (d, e) Quantitative analysis of the BMS score and incline angle at 28 dpi from (b) and (c), respectively ( $n=6$ per group). (f) H6istological assessment of the injured spinal cord by HE staining at 7 dpi. (g) Nissl staining of the injured spinal cord to evaluate the neuronal survival in each group at $7 \mathrm{dpi}$. Results are represented as mean \pm SEM. ${ }^{\#} P<0.05,{ }^{\#} P<0.01,{ }^{\# \# \#} P<0.001$, and ${ }^{\# \# \#} P<0.0001$.

with cresyl violet acetate for Nissl body staining according to the instructions. After staining, the slices were successively dehydrated and transparentized using 95\% alcohol for $2 \mathrm{~min}$ and xylene for $5 \mathrm{~min}$, respectively. Finally, the film was sealed with neutral resin. The damage area was photographed by an optical microscope (Olympus, Tokyo, Japan).

2.8. Locomotor Function Recovery Assessment. The hind limb locomotor function of mice was evaluated by the Basso Mouse Scale (BMS) scoring [30] and inclined plane test at 0, 1, 3, 7, 14,21 , and 28 days after injury (dpi). In short, animals were allowed to move freely a period of time in an empty room, and the BMS scores of mice were recorded according to the observation of paw posture, posterior ankle joint mobility, trunk stability, tail posture, and coordination. The BMS score of mice ranges from 0 (completely paralysis) to 9 (normal locomotion). For the inclined plane test, mice were placed on a rubber board, and then, the angles at which the mice could not hold its position for $5 \mathrm{sec}$ were defined as the maximum angles, which could be used to evaluate the hind limb strength of mice [31]. The results of mouse hind limb movements were blindly recorded by two trained investigators.

2.9. ELISA. BV2 cells were coincubated with LPS combined with or without sesamol. IL- 6 and TNF- $\alpha$ produced from cells were detected by using a commercial ELISA kit according to the instructions. The OD values at $450 \mathrm{~nm}$ and $630 \mathrm{~nm}$ were determined by using the SpectraMax microplate reader.

2.10. Statistical Analysis. Results are expressed as the mean \pm SEM. The significant difference comparison of two groups was carried out by two-tailed Student's $t$-test. The statistical significance comparison of multiple groups was conducted by one-way analysis of variance (ANOVA) test with Tukey's multiple comparison test. All statistical analyses were conducted by using GraphPad Prism 8 software. $P<0.05$ was regarded significant.

\section{Results}

3.1. Sesamol Improves Locomotor Functional Restoration in Mice after SCI. The BMS score and inclined plane test were applied to assess locomotor functional restoration, and HE staining as well as Nissl staining was carried out to evaluate histological outcomes in mice suffering from SCI. Prospec- tively, BMS scores of both injury groups were distinctly lower than that of the sham group, while injured mice treated with sesamol exhibited a distinct amelioration of posterior limb motor function with higher BMS scores and better coordinated crawling at 14,21 , and 28 dpi relative to mice suffering from SCI alone (Figures $1(\mathrm{~b})$ and 1(c)). Cavity of necrotic tissue was detected by HE staining, and neuronal survival was measured using Nissl staining at $7 \mathrm{dpi}$, respectively. Result of HE staining displayed that sesamol treatment decreased SCI-induced cavity of necrotic tissue (Figure 1(f)). Similar to the result, sesamol reduced SCIinduced neuron loss; in other words, dramatically more survival neurons existed in the sesamol-treated group than in mice suffering from SCI alone detected by Nissl staining (Figure 1(g)). Collectively, the above data indicate that sesamol has a neuroprotective role in locomotor function recovery in mice after SCI.

3.2. Sesamol Attenuates Neuronal Apoptosis following SCI. To confirm whether sesamol could reduce apoptosis induced by SCI, the level of apoptosis was measured by western blot and immunofluorescence at $7 \mathrm{dpi}$. Our results showed that traumatic SCI upregulated cleaved caspase-3 and Bax (markers of proapoptotic proteins) protein expression and weakened the protein level of $\mathrm{Bcl}-2$ (an antiapoptotic protein), which were dramatically reversed by sesamol administration (Figures 2(a)-2(d)). These above data were confirmed by immunofluorescence staining of NeuN (marker for neuron) and cleaved caspase-3. Similarly, SCI increased cleaved caspase-3-positive neurons, but that was decreased by sesamol (Figure 2(e)). These results indicate that sesamol can prevent neuron apoptosis after SCI in vivo.

3.3. Sesamol Reduces Proinflammatory Cytokine Release and Regulates Phenotypic Polarization of Microglia. Accumulating evidences have revealed that inflammatory responses play key roles in secondary SCI. Therefore, levels of IL-6 and TNF- $\alpha$ were determined using western blot in vivo at $3 \mathrm{dpi}$ and by ELISA in LPS-mediated BV2 cells. Compared with uninjured mice, acute SCI induced dramatic increase protein level of IL- 6 and TNF- $\alpha$ in the damaged spinal cord, while the level of cytokines was significantly decreased by sesamol (Figures 3(a)-3(c)). Consistent with the result, 


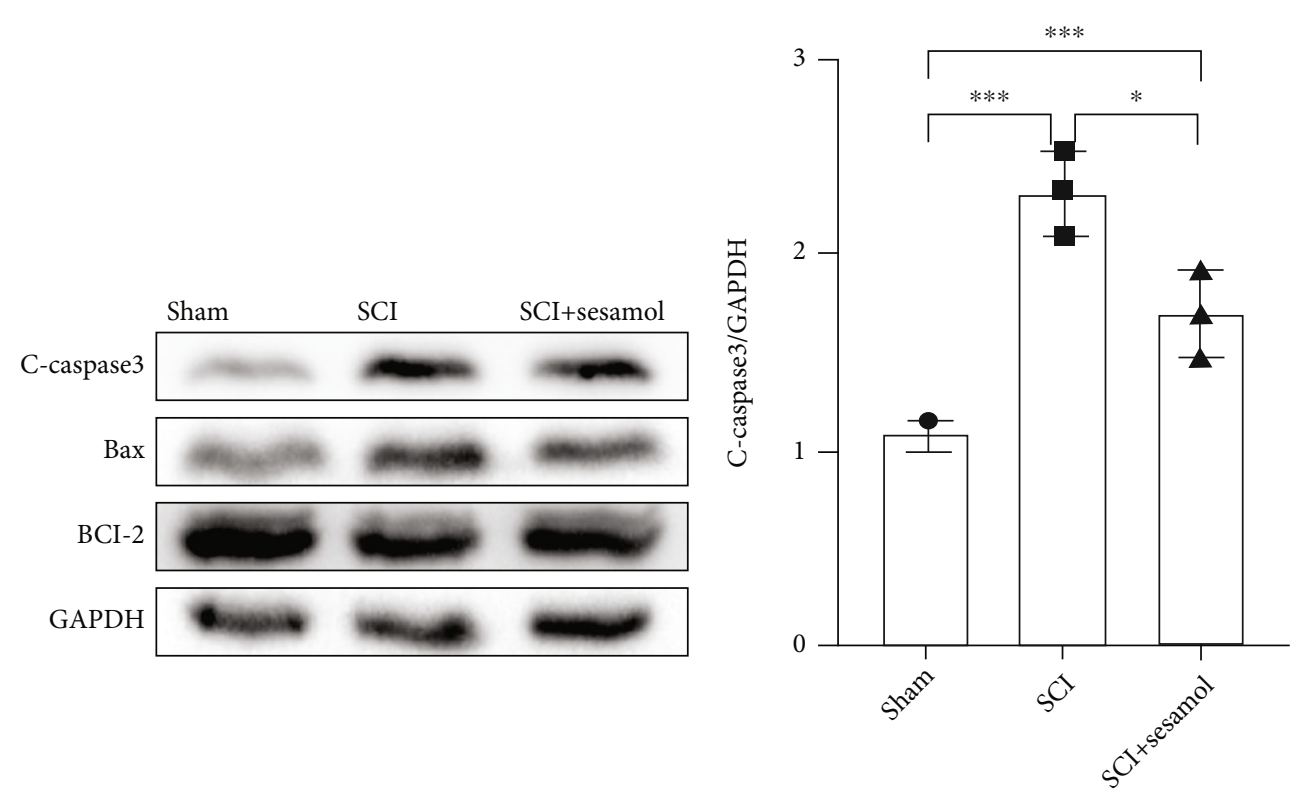

(a)

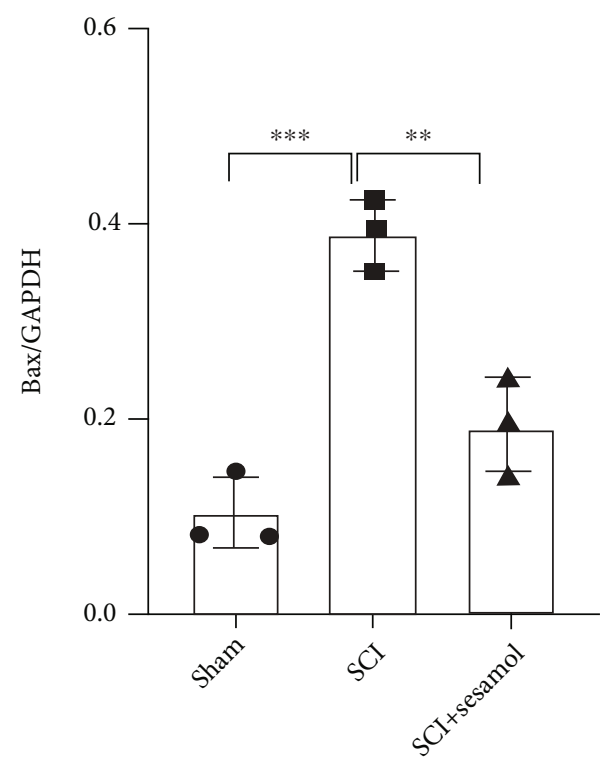

(c)

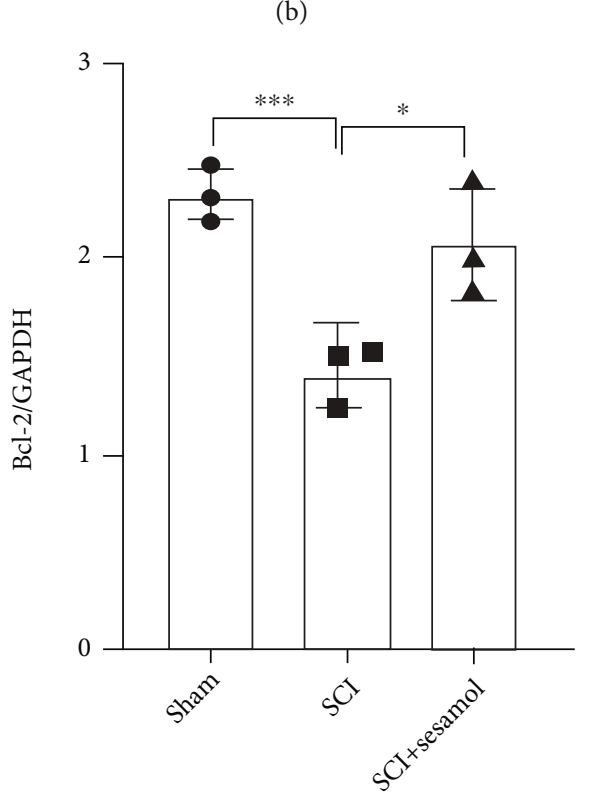

(d)

FIGURE 2: Continued. 


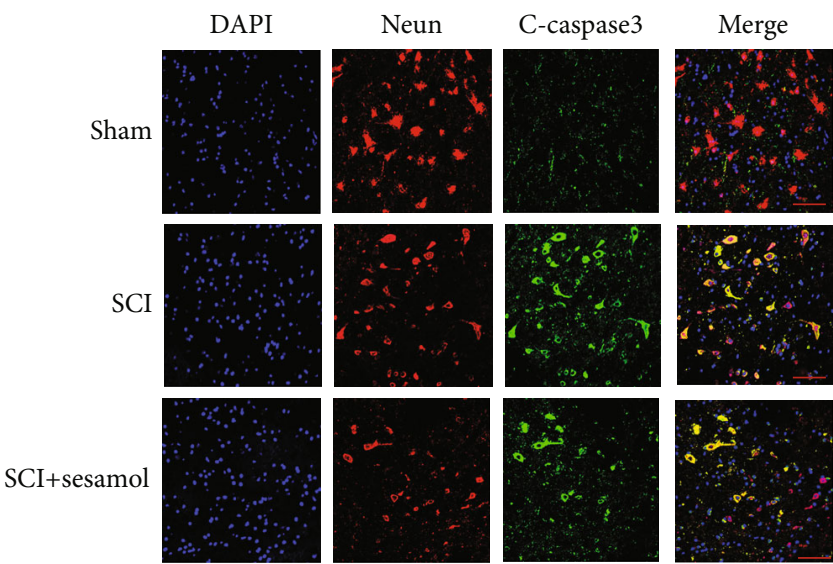

(e)

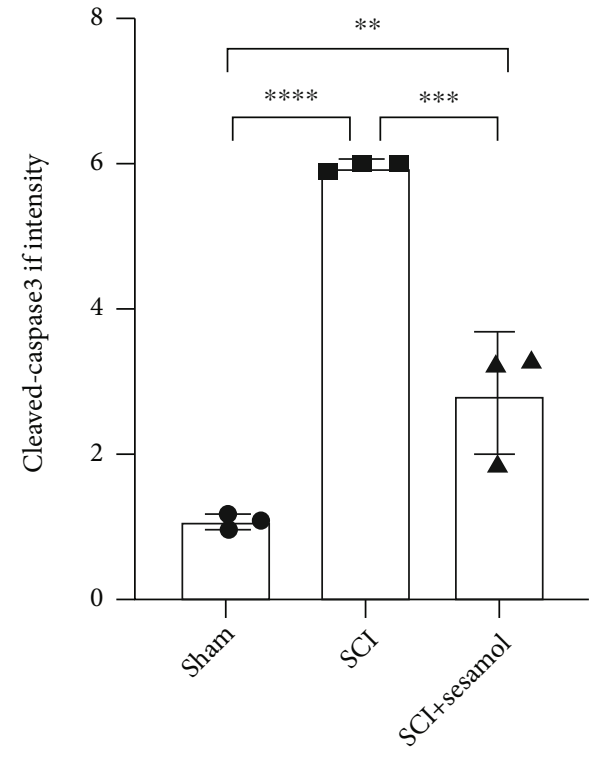

(f)

FIgURE 2: Sesamol protects neurons from apoptosis in mice after SCI. (a) Western blot images exhibiting apoptosis-related protein levels in the injured spinal cord of mice from each group at $7 \mathrm{dpi}$. (b-d) Quantitative analysis of the expression these proteins from (a) normalized to GAPDH. $n=3$ per group. (e) Sesamol reduced the fluorescence degree of cleaved caspase-3 (green) in neurons (established by NeuN, red), and DAPI (blue) is for the nuclear staining (scale bar $=50 \mu \mathrm{m}$ ). (f) Quantitative analysis of the fluorescence degree of cleaved caspase-3. Results are expressed as mean \pm SEM ( $n=3$ per group). ${ }^{\# \#} P<0.05,{ }^{\# \# \#} P<0.001$, and ${ }^{\# \# \# \# ~} P<0.0001$.

increase in TNF- $\alpha$ and IL- 6 was also distinctly overturned by sesamol in LPS-induced BV2 cells (Figures 3(d) and 3(e)).

To further understand the anti-inflammation role of sesamol in mice after SCI, phenotypic polarization of microglia was determined at $3 \mathrm{dpi}$. The data obtained by western blot analysis showed that sesamol administration prevented M1 microglia-related protein (CD86) expression and increased protein levels of M2 microglial mediators, including CD206 and arginase 1 (Arg1) (Figures 3(a) and 3(f)-3(h)). Consistent with the results, immunofluorescence analysis also verified that sesamol improved the CD206 protein expression in $\mathrm{IBA}^{+}$microglia after SCI (Figure 3(i)). These above data indicate that sesamol inhibits production of proinflammatory factors and induces M2 phenotype microglial polarization.

\subsection{Effect of Sesamol on AMPK/SIRT1/NF- $\kappa B$ Pathways in} SCI. To understand the underlying mechanism of sesamol alleviating inflammation and promoting SCI restoration, effects of sesamol on regulating AMPK/SIRT1/NF- $\kappa$ B pathways at $3 \mathrm{dpi}$ were evaluated. Compared with uninjured mice, SCI resulted in significant decreases in SIRT1 protein expression and the $\mathrm{p}$-AMPK/AMPK ratio, which was reversed by sesamol treatment (Figures 4(a)-4(c)). Activation of NF- $\kappa \mathrm{B}$ is connected with proinflammatory factor secretion and phenotypic changes of microglia [32]; thus, protein level of NF- $\kappa \mathrm{B}$ p 65 and its phosphorylation level (p-p65) were determined by western blot analysis. The result exhibited that the ratio of p-p65/p65 was remarkably increased in mice following SCI relative to uninjured mice, which was dramatically inhibited by sesamol administration
(Figures 4(a) and 4(d)). Similar to the result, SIRT1 was upregulated and P65 was downregulated in $\mathrm{Iba}^{+}$microglia after SCI with sesamol administration (Figures $4(\mathrm{e})$ and $4(\mathrm{f}$ )). These above data indicate that sesamol may activate AMPK/SIRT1 and suppress activation of NF- $\kappa$ B pathways in mice after SCI.

3.5. AMPK Participates in Sesamol Alleviating Inflammation in LPS-Stimulated Microglia. To further confirm whether sesamol inhibited inflammation by activating the AMPK pathway following SCI, a specific AMPK inhibitor (compound C) was applied in LPS-induced BV2 cells. Then, protein expression of proinflammatory cytokines was examined by western blot and ELISA. Results in Figures 5(a)-5(e) showed that sesamol $(10 \mu \mathrm{M})$ significantly reduced the protein expression of IL- 6 and TNF- $\alpha$ in LPS-induced BV2 cells, which was reversed by compound $\mathrm{C}(5 \mu \mathrm{M})$. Collectively, these results reveal that AMPK may participate in antiinflammation of sesamol in microglia stimulated by LPS.

3.6. AMPK Activation Participates in Sesamol-Induced M2 Microglial Polarization. M1 phenotype microglia have been proven to be neurotoxic and induce inflammation response, while M2 microglia perform anti-inflammation. In this study, western blot and immunofluorescence were applied to further determine whether AMPK activation participates in sesamol-induced M2 microglial polarization in vitro. The results showed that both CD86 (a M1 microglia indicator) and CD206 (an established marker for M2 microglia) were significantly increased in LPS-stimulated BV2 cells. However, sesamol administration decreased dramatically 


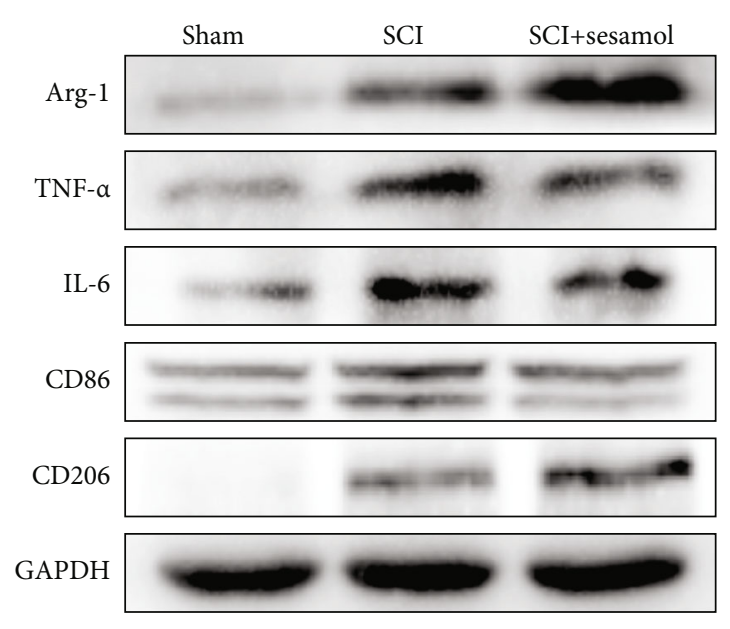

(a)

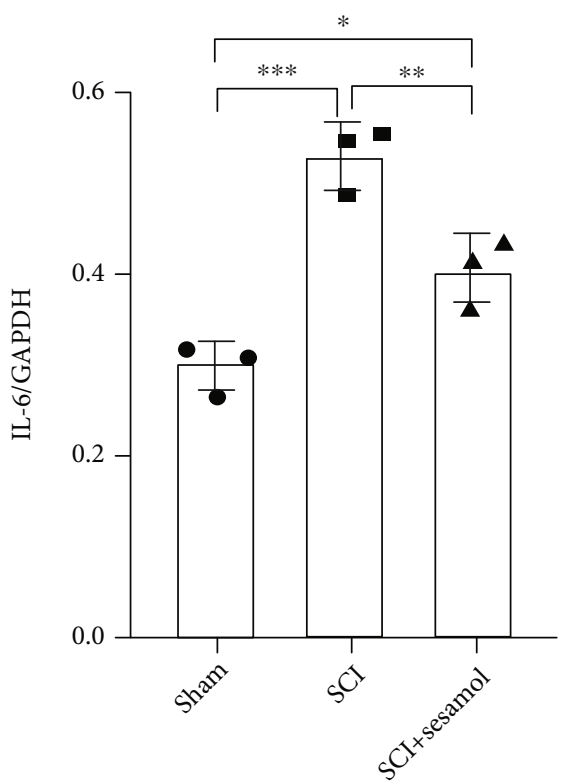

(b)

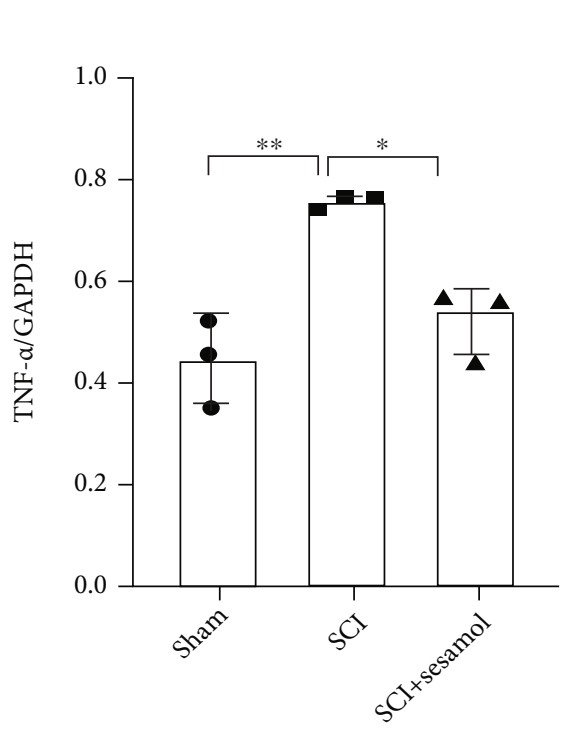

(c)

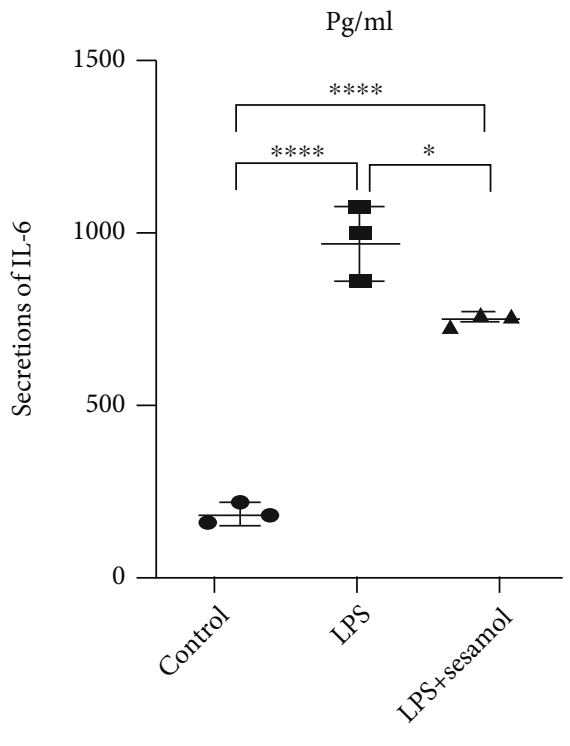

(d)

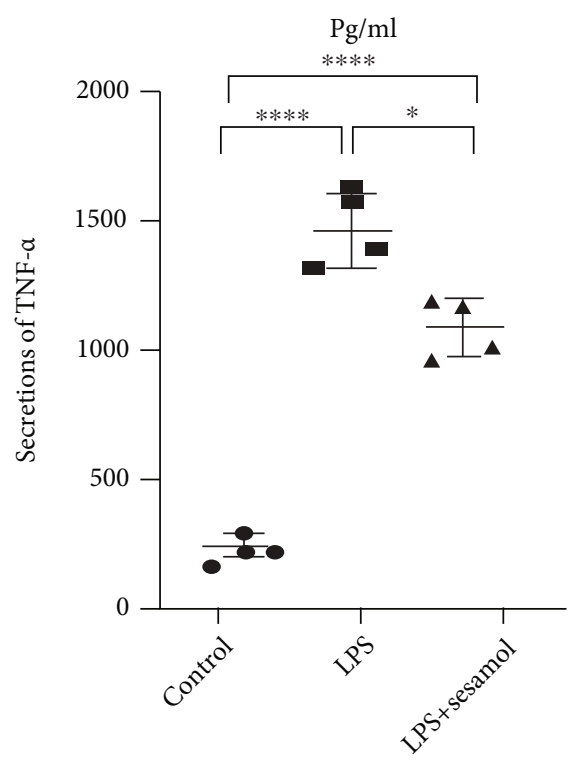

(e)

Figure 3: Continued. 


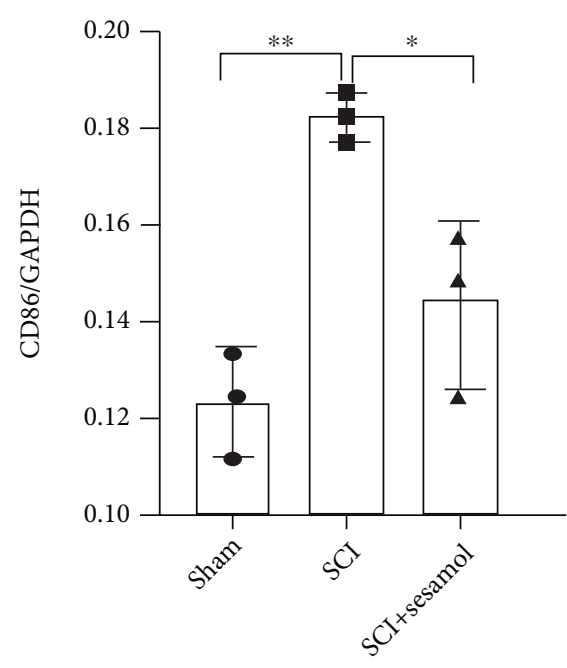

(f)

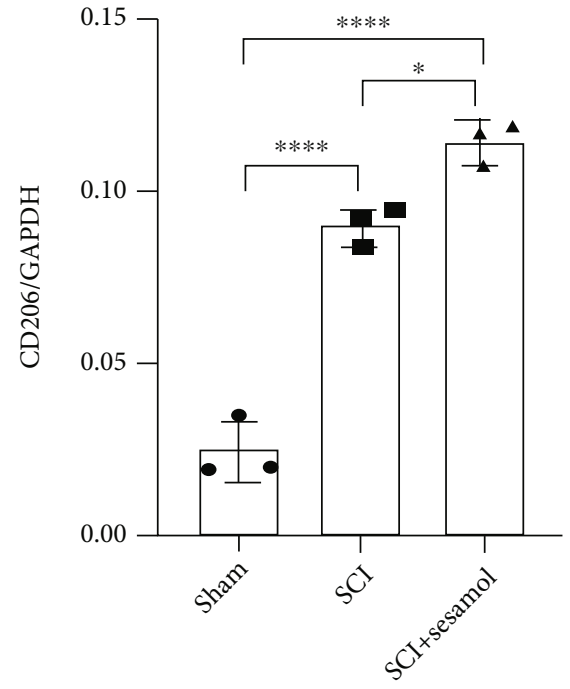

(g)

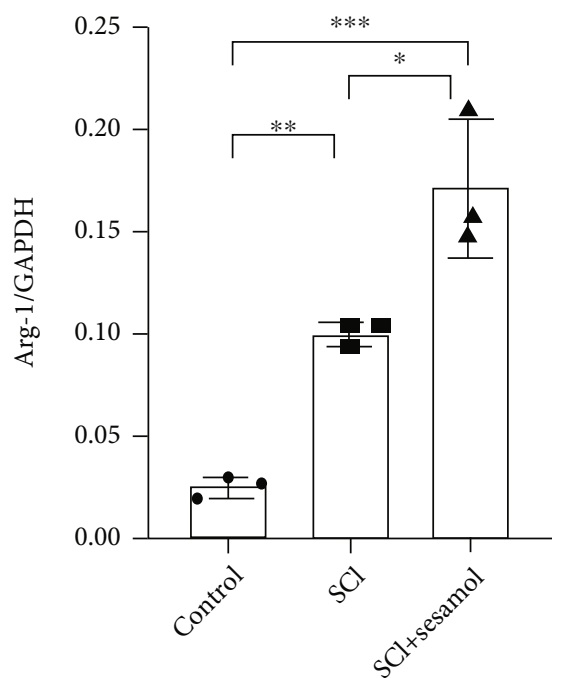

(h)

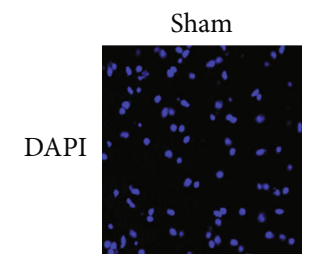

SCI

SCI+sesamol
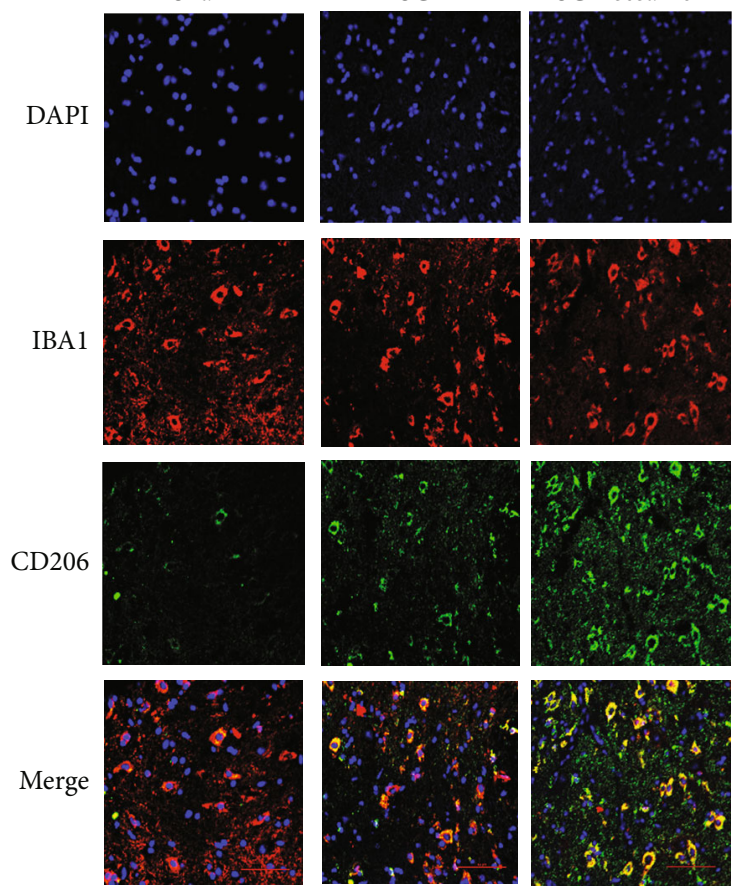

(i)

FIgURE 3: Sesamol attenuates inflammation and induces M2 phenotype microglial polarization in mice suffering from SCI. (a) Western blot images showing protein levels of IL-6, TNF- $\alpha$, CD86, Arg-1, and CD206 in the injured spinal cord of mice at 3 dpi. (b, c) Quantification of TNF- $\alpha$ and IL- 6 from (a), respectively $(n=3)$. (d, e) Statistical analysis of IL- 6 and TNF- $\alpha$ concentrations determined by ELISA in BV 2 cells suffering from LPS ( $n=4$ per group). ( $\mathrm{f}-\mathrm{h})$ The quantitative analysis of CD86, CD206, and Arg-1 from (a) ( $n=3$ per group). (i) Sesamol increased the fluorescence degree of CD206 (green) in activated microglia (established by IBA1, red), and DAPI (blue) is for the nuclear staining $($ scale bar $=50 \mu \mathrm{m})$. Values are represented as the mean \pm SEM. ${ }^{\#} P<0.05,{ }^{\# \#} P<0.01,{ }^{\# \# \#} P<0.001$, and ${ }^{\# \# \# \#} P<0.0001$.

CD86 protein level and further induced CD206 protein expression, which were significantly reversed by the AMPK inhibitor compound C (Figures 6(a)-6(e)). Consistent with the result, fluorescence intensity of CD206 in LPS-mediated BV2 cells was also remarkably increased by sesamol treatment, which was notably reduced by compound C (Figure 6(f)). These results confirm that sesamol promotes $\mathrm{M} 2$ polarization in BV2 cells partly regulated by the AMPK pathway.
3.7. Sesamol Alleviating Inflammation Response in Microglia Is Regulated via the AMPK/SIRT1/NF- $\kappa B$ p65 Pathway. Evidences have shown that SIRT1 is a downstream target of the AMPK signaling pathway and participates in antiinflammation via suppression of NF- $\kappa$ B p65 activation [23-25]. To further verify sesamol playing an antiinflammatory role in microglia via the AMPK/SIRT1/NF$\kappa \mathrm{B}$ pathway, compound $\mathrm{C}$ was applied in BV2 cells, and 


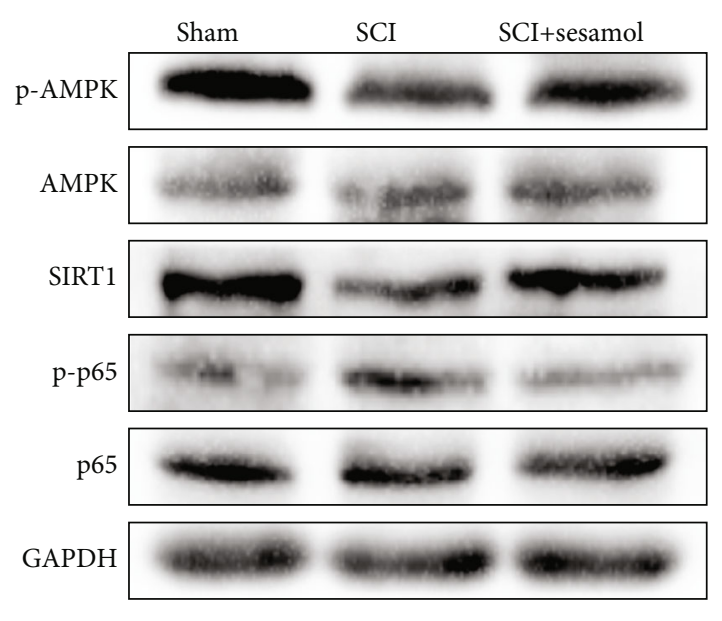

(a)

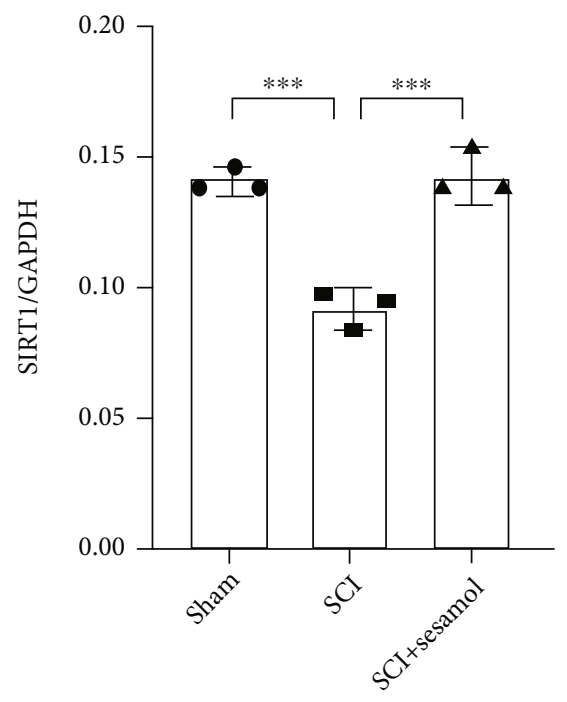

(c)

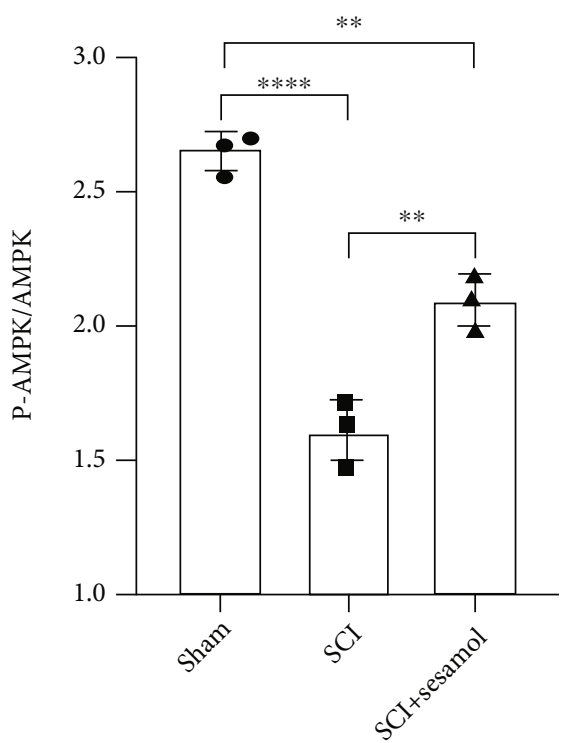

(b)

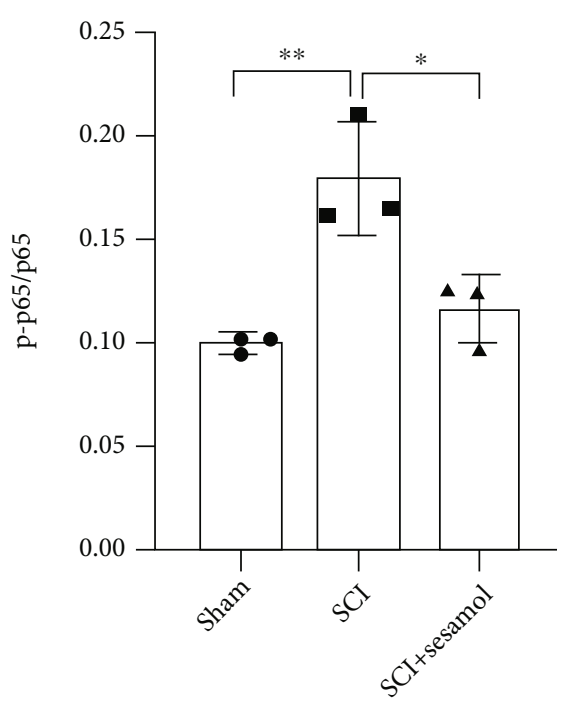

(d)

Figure 4: Continued. 


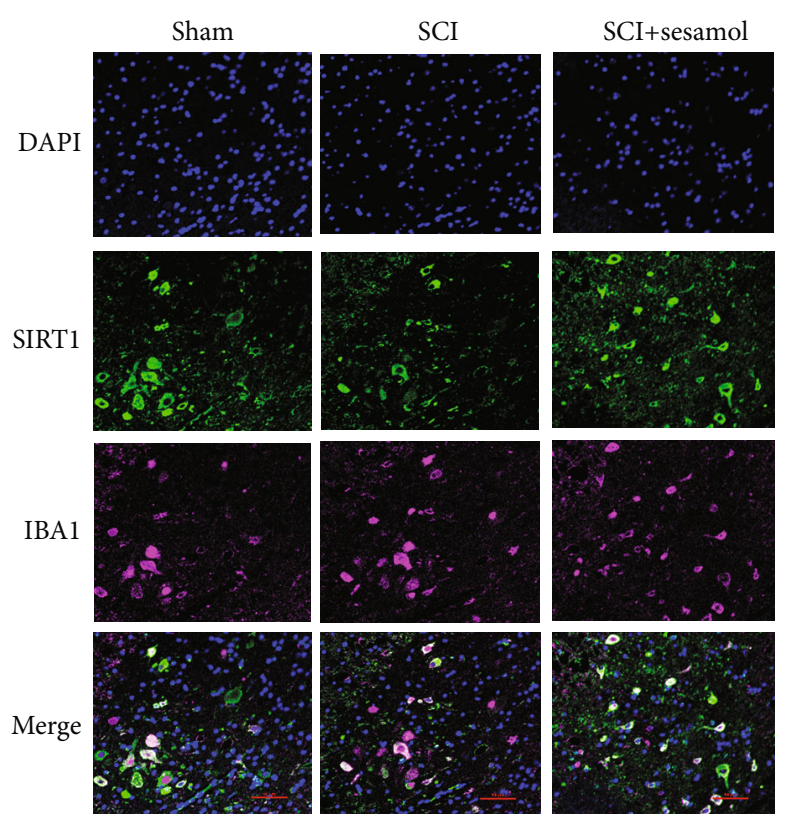

(e)

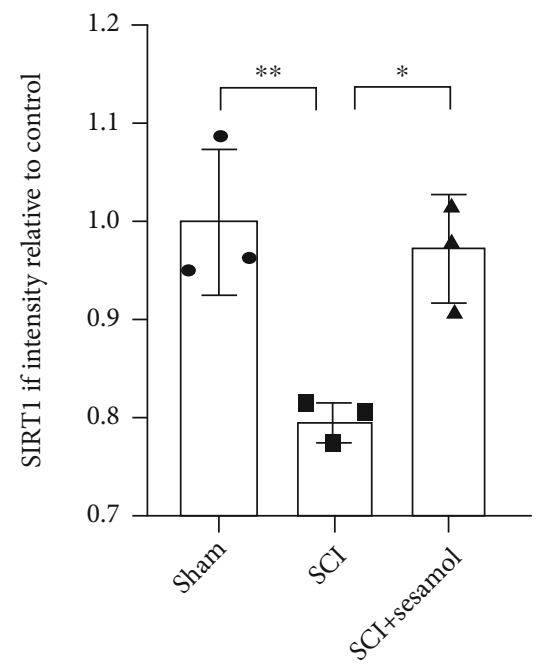

(g)
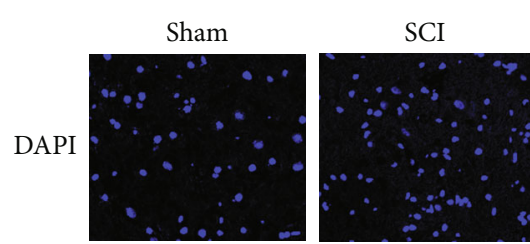

SCI+sesamol
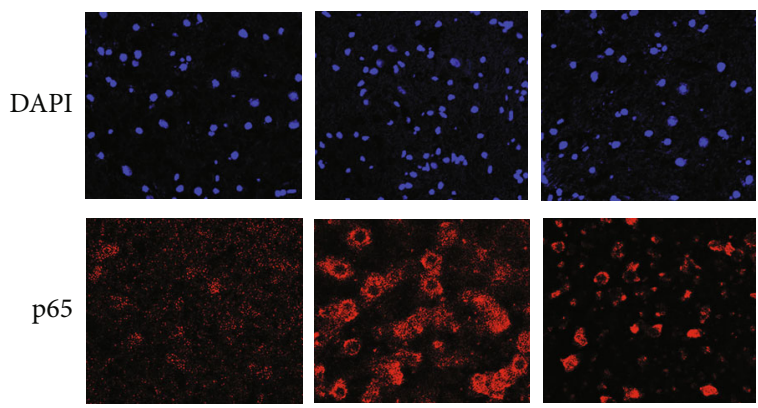

IBA1
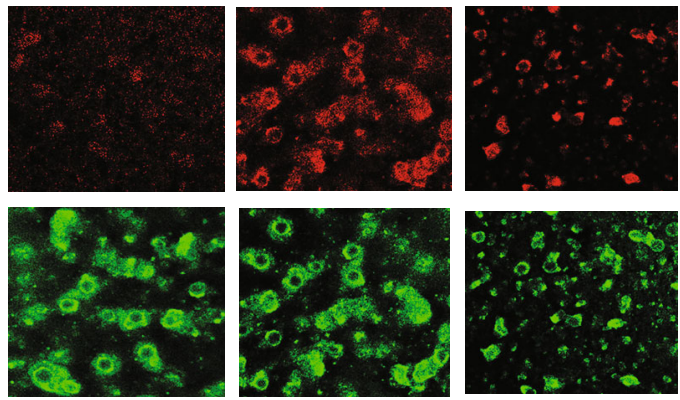

Merge
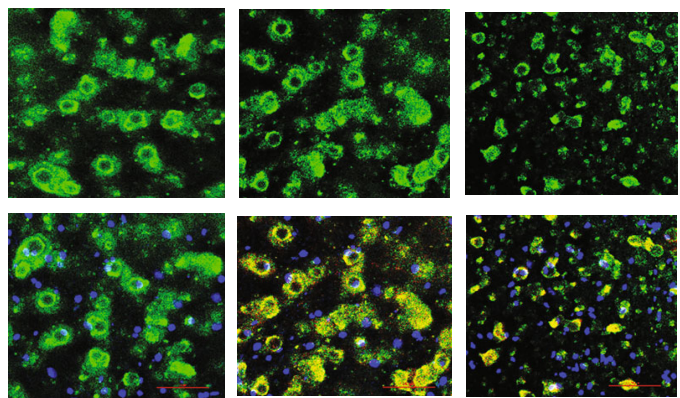

(f)

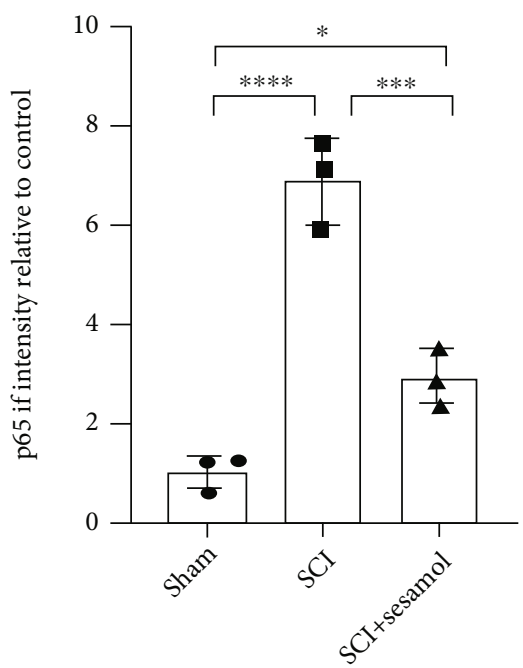

(h)

FIGURE 4: Sesamol regulates AMPK/SIRT1/NF- $\kappa$ B pathways in mice following SCI. (a) Western blot images showing protein expressions of p-AMPK, AMPK, SIRT1, p-p65, and p65 in the spinal cord of mice from uninjured, SCI, and sesamol-treated group at 3 dpi. (b-d) Quantitative analysis of western blot results from (a) ( $n=3$ per group). (e) Representative images showing that sesamol increased the fluorescence degree of SIRT1 (green) in activated microglia (established by IBA1, purple), and DAPI (blue) is for the nuclear staining (scale bar $=50 \mu \mathrm{m})$. (f) Sesamol reduced the fluorescence degree of p65 (red) in activated microglia $(\mathrm{scale} b a r=50 \mu \mathrm{m})$. $(\mathrm{g}$, $\mathrm{h})$ Quantification of the fluorescence degree of SIRT1 and p65 in activated microglia from (e) and (f), respectively. Data are represented as the mean \pm SEM $\left(n=3\right.$ per group). ${ }^{\#} P<0.01,{ }^{\# \#} P<0.001$, and ${ }^{\# \# \#} P<0.0001$.

then, protein expression of SIRT1, AMPK, and NF- $\kappa$ B was analyzed using western blot. Results from Figures $7(\mathrm{a})-$ 7(d) showed that the SIRT1 protein level and p-AMPK/ AMPK ratio increased significantly while the p-p65/p65 ratio reduced remarkably in sesamol-treated BV2 cells compared with cells exposed to LPS only. However, these trends caused by sesamol were apparently overturned by compound C. Moreover, sesamol also resulted in distinct enhancement of fluorescence intensity of SIRT1 and a remarkable reduction of p65 fluorescence intensity, which were reversed by compound C (Figures 7(e) and 7(f)). Combined with data from Figure 5, these data further confirm that sesamol prevents inflammation through regulation of the AMPK/SIRT1/NF- $\kappa$ B pathway.

\section{Discussion}

Secondary SCI is accompanied by long-term neuroinflammation, oxidative stress, and apoptosis [33]. Sesamol is a kind of excipient used in food and medicine and plays roles 


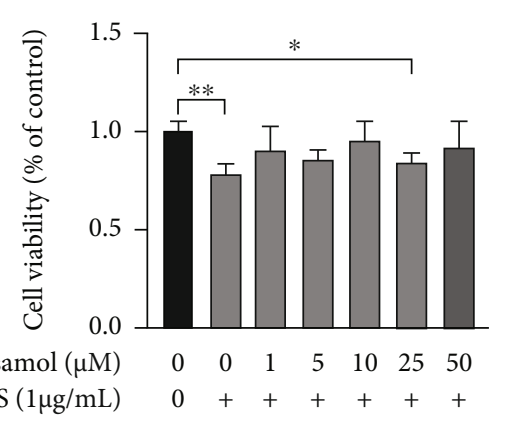

(a)

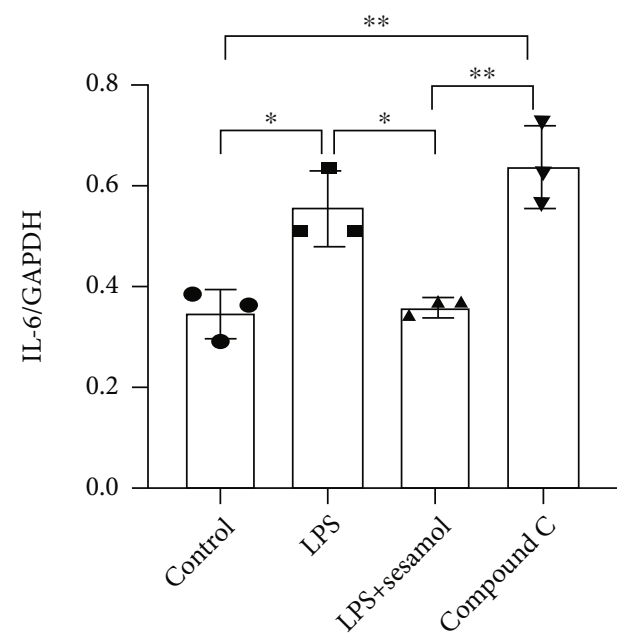

(c)

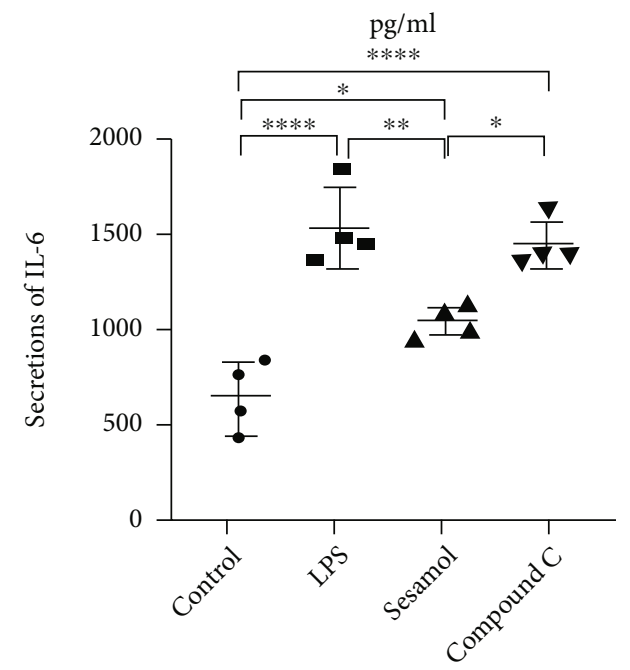

(e)

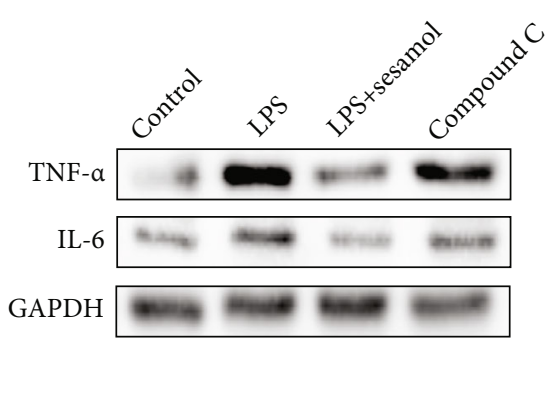

(b)

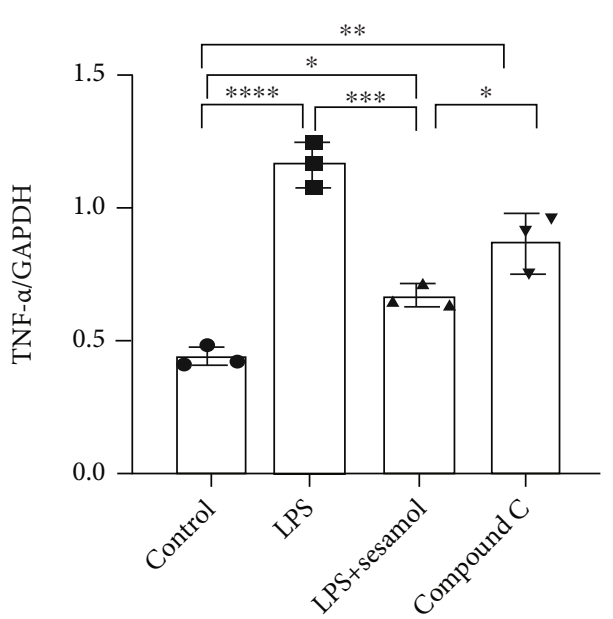

(d)

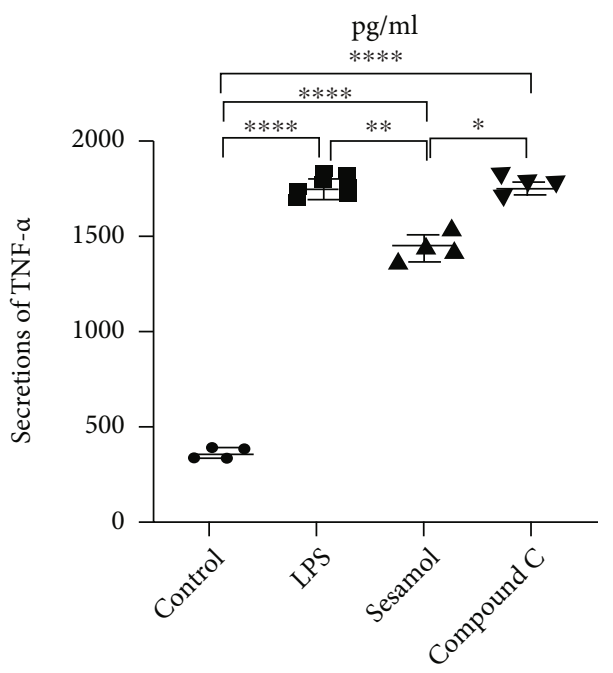

(f)

FiguRE 5: Sesamol-reduced proinflammatory cytokine secretions are reversed by compound C in LPS-mediated BV2 cells. (a) Quantification cell viability of BV2 cells incubated with LPS or (and) sesamol determined by using the CCK-8 kit. (b) Western blot images showing protein expressions of TNF- $\alpha$ and IL-6 in BV2 cells exposed to different conditions. (c, d) Quantitative assessment of TNF- $\alpha$ and IL-6 from (a), respectively ( $n=3$ per group). (e, f) Statistical analysis of IL- 6 and TNF- $\alpha$ concentrations determined using ELISA in BV2 cells suffering

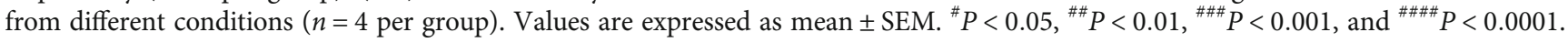

in antioxidant stress, antiaging, and anti-inflammation [34-36]. Nevertheless, the protective roles and the underlying mechanism of sesamol in SCI repair remain poorly understood. The current study examined the neuroprotec- tive, antineuroinflammatory, and antiapoptotic roles of sesamol in mice following SCI and further explored the underlying mechanisms both in mice and in BV2 cells. Our results indicate that sesamol can ameliorate neuronal 


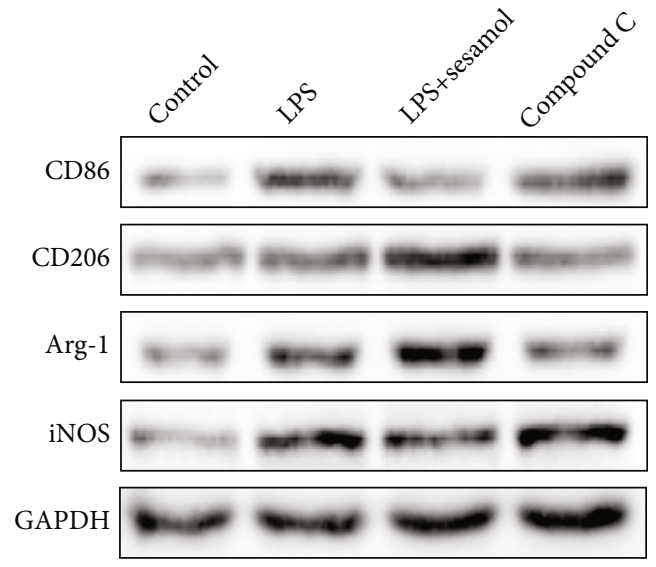

(a)

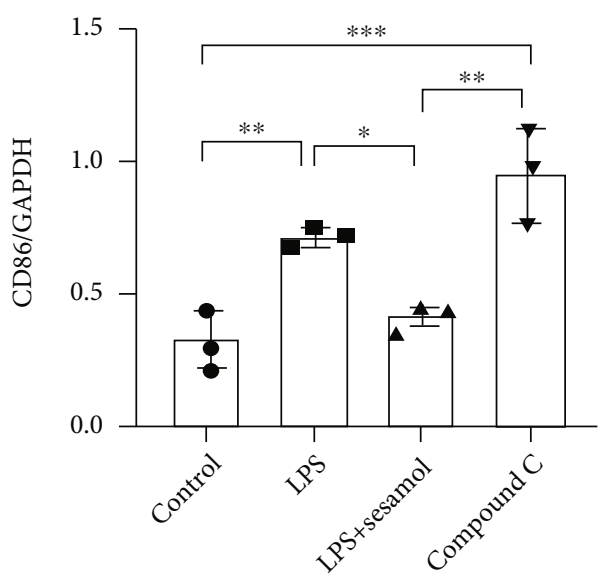

(b)

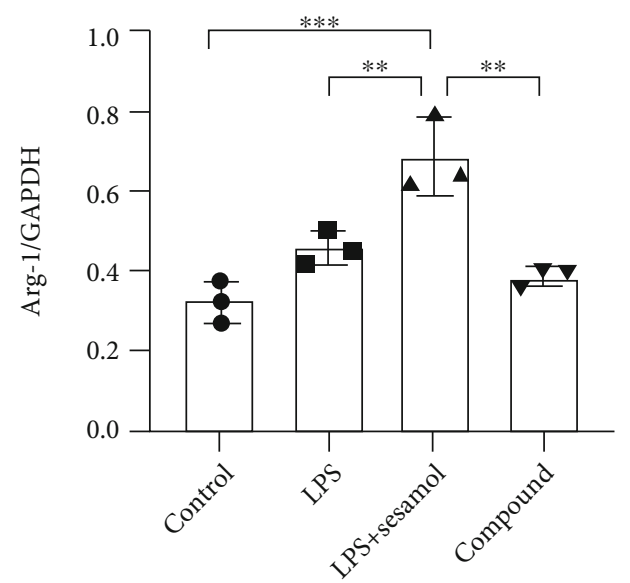

(d)

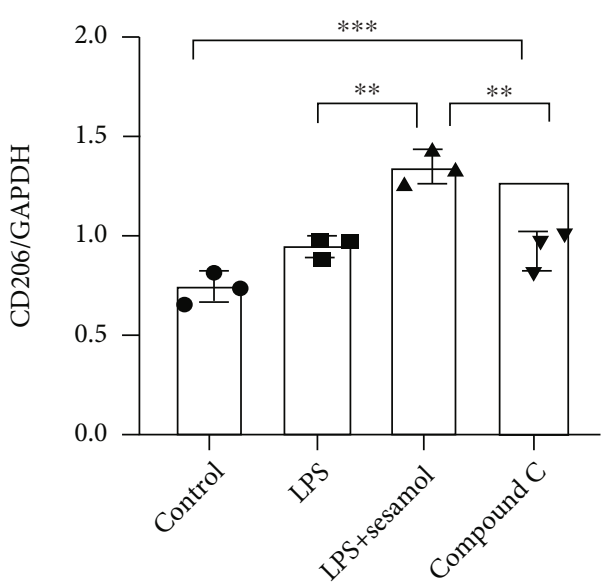

(c)

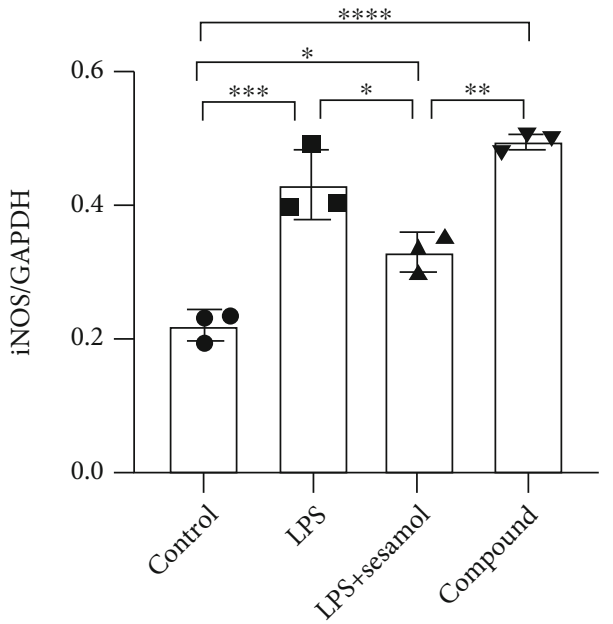

(e)

Figure 6: Continued. 


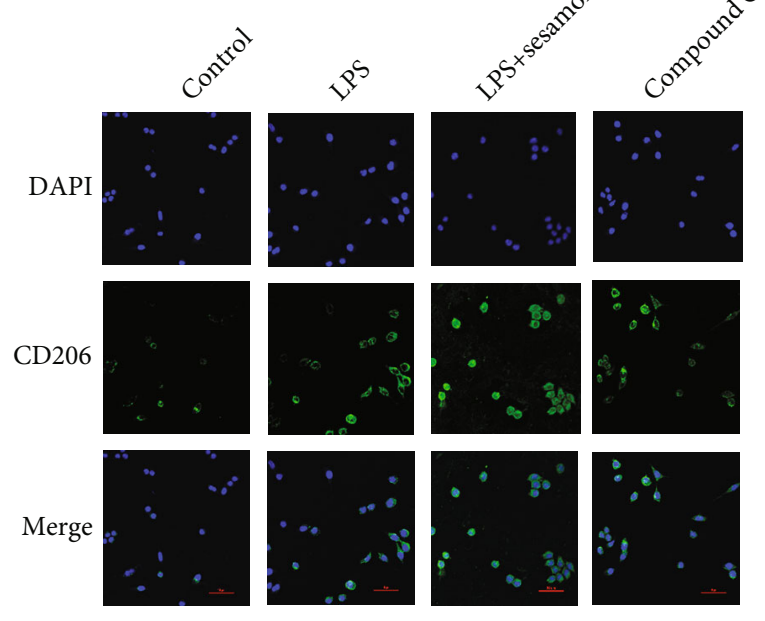

(f)

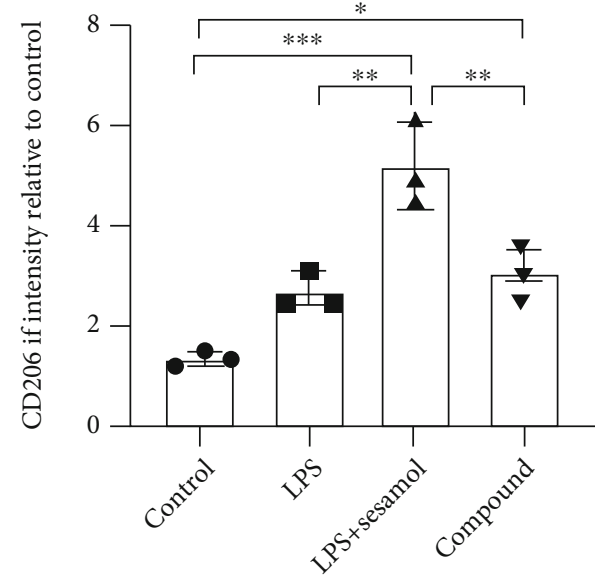

(g)

Figure 6: AMPK participates in sesamol-stimulated M2 microglial polarization. (a) Western blot images showing that compound C reversed effects of sesamol on protein levels of CD86, iNOS, Arg-1, and CD206 in BV2 cells. (b-e) Quantitative analysis of CD86, CD206, Arg-1, and iNOS from (a) normalized to GAPDH. (f) Compound C overturned fluorescence intensity of CD206 (green) increased by sesamol in microglia (scale bar $=50 \mu \mathrm{m})$. (g) Quantification of the fluorescence degree of CD206 from (f). Results are

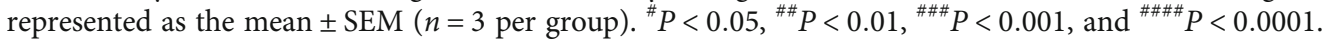

apoptosis, reduce inflammatory response, promote $\mathrm{M} 2$ microglial polarization, and improve neurological function restoration in mice after SCI. Furthermore, our data provide mechanistic evidence for the hypothesis that the AMPK/ SIRT1/NF- $\kappa$ B pathway might be important to the antineuroinflammatory ability of sesamol in microglia.

SCI leads to direct or indirect damage to neurons, causing rampant neuronal apoptosis at early stages of injury, which is one of key obstacles to the SCI restoration. Thus, preventing neuron against apoptosis is believed to be an effective approach to promote neural restoration after SCI. In the current study, sesamol could promote locomotor functional recovery and histological outcomes in mice after SCI. Our further results demonstrated that sesamol caused dramatic reduction of cleaved caspase- 3 and Bax but a significant upregulation of $\mathrm{Bcl}-2$, particularly within neurons in mice after SCI. Ample evidences indicate that Bax and cleaved caspase- 3 participate in proapoptosis, while $\mathrm{Bcl}-2$ has an antiapoptotic role $[29,37]$. This work provides novel evidence that sesamol plays an important role of antiapoptosis within neurons in mice suffering from SCI.

Immune response is activated after $\mathrm{SCI}$, and $\mathrm{NF}-\kappa \mathrm{B}$ (p65) is one of the important regulators participating in infiltration of monocytes, neutrophils, and activated microglia into the damaged site and secretion of a lot of proinflammatory cytokines, including IL- 6 and TNF- $\alpha$ [38, 39]. Ample evidences have demonstrated that microglia in CNS contribute to neuroinflammation progress [40, 41]. Our results revealed that sesamol significantly reduced TNF- $\alpha$ and IL-6 protein levels in mice suffering from SCI and in LPSmediated microglia. This is consistent with the previous study showing that sesamol inhibits dextran sulphate sodium-induced inflammation in colitis mice [35]. After CNS injury, microglia are activated and polarized to the M1 phenotype with proinflammatory function and the M2 phenotype with anti-inflammatory effect $[42,43]$. However, whether sesamol alleviates neuroinflammation related to regulating polarization of the microglial phenotype is still unknown. In our study, the protein level of CD86, an indicator of M1 microglia, was significantly increased after SCI and then dramatically reduced after sesamol administration. In contrast, the protein levels of Arg-1 and CD206 specially expressed in M2 microglia were increased at early days after SCI and further increased after sesamol administration. These data indicate that sesamol treatment decreases the proportion of M1 but increases M2 phenotype cells in activated microglia, which may contribute to antineuroinflammation.

Sesamol is extracted from sesame oil and can be used as an antioxidant in foods and medicines [44]. Several studies have revealed that sesamol protects the body from various disease conditions such as obesity, hyperlipidemia, and diabetic foot ulcer mainly through regulating lipid as well as energy metabolism and reducing inflammatory cell infiltration [45-47]. Ample previous studies have also documented that sesamol has anti-inflammatory effects via upregulating AMPK signaling and inhibiting NF- $\kappa$ B activation [26, 27]. Moreover, sesamol can activate SIRT1 signaling to attenuate oxidative stress which will lead to cell apoptosis [28]. It is well known that AMPK is a key energy sensor that mediates cellular energy balance in the body [48]. AMPK also is found to be an important regulator participating in the 


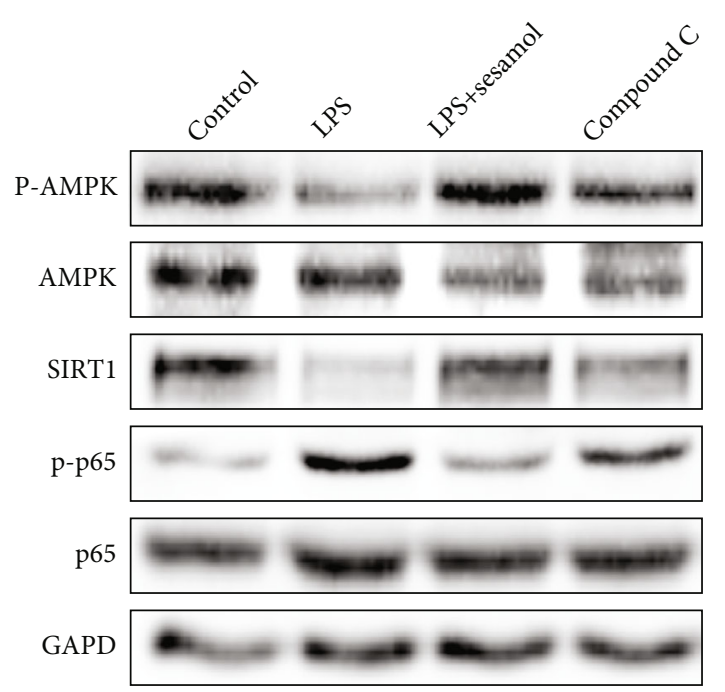

(a)

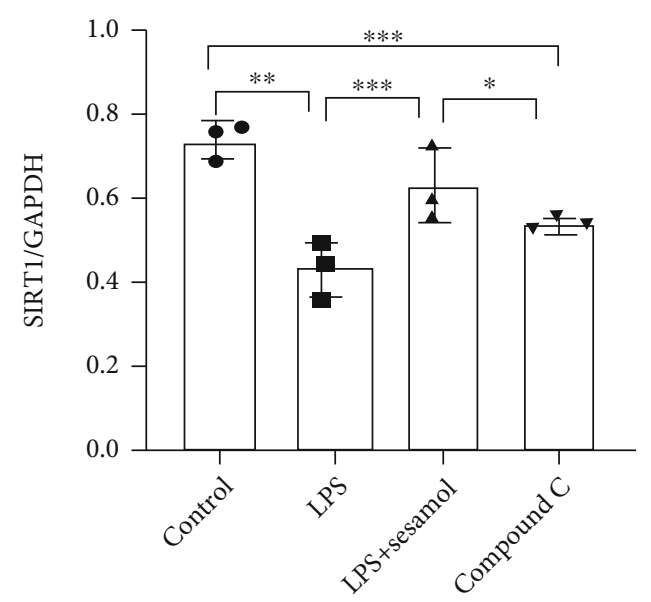

(c)

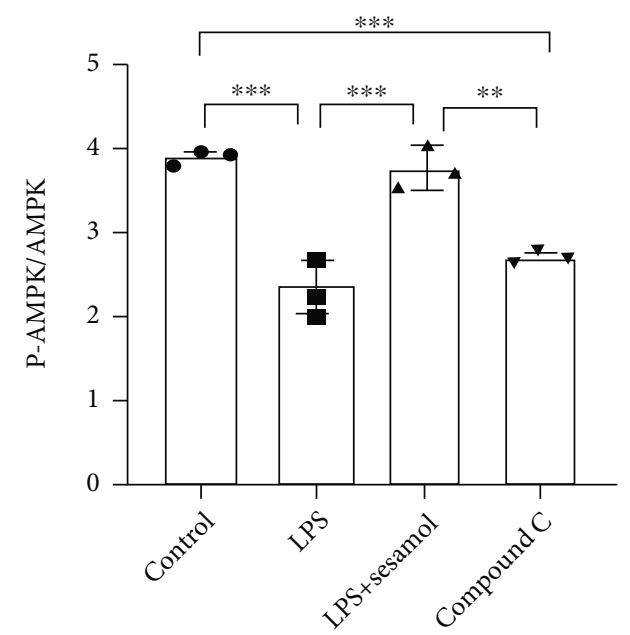

(b)

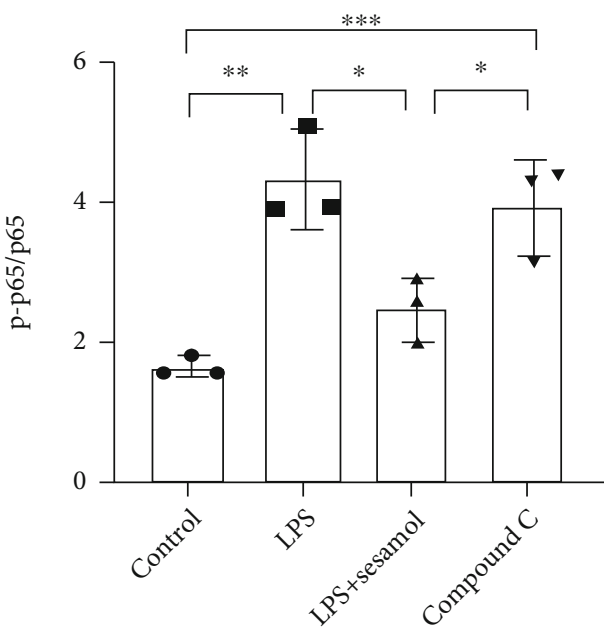

(d)

Figure 7: Continued. 


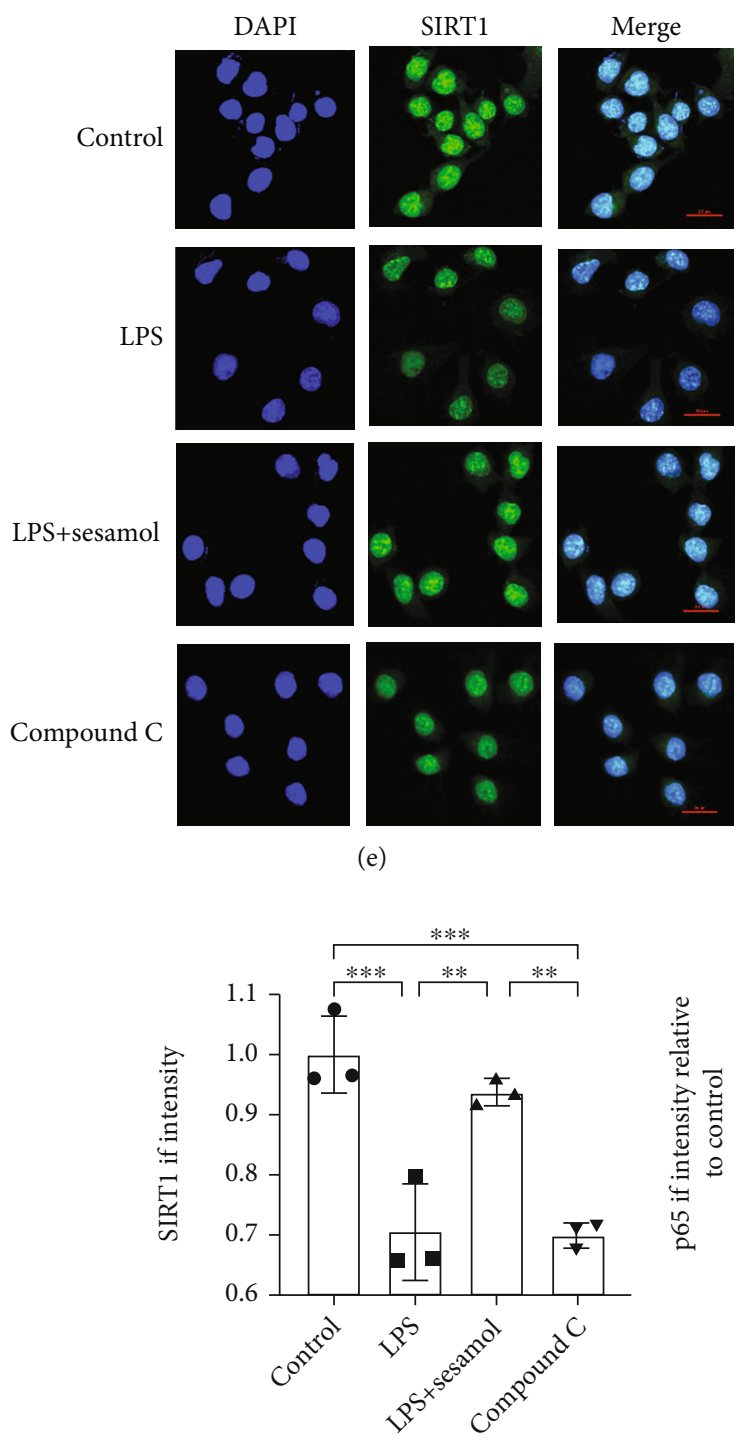

(g)

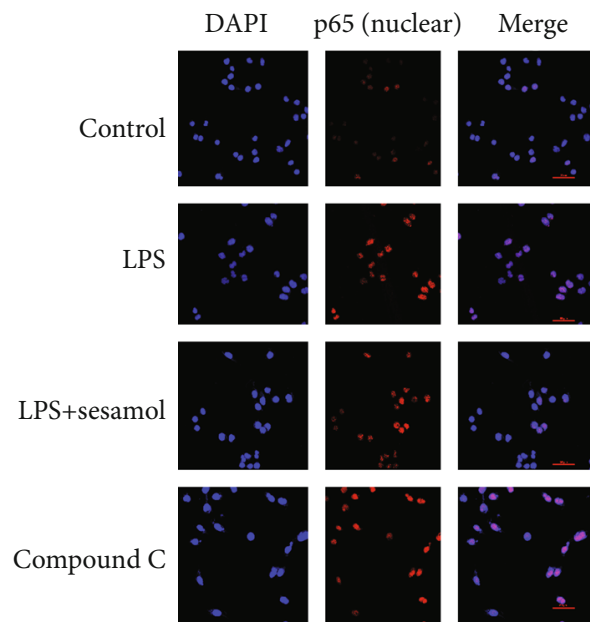

(f)

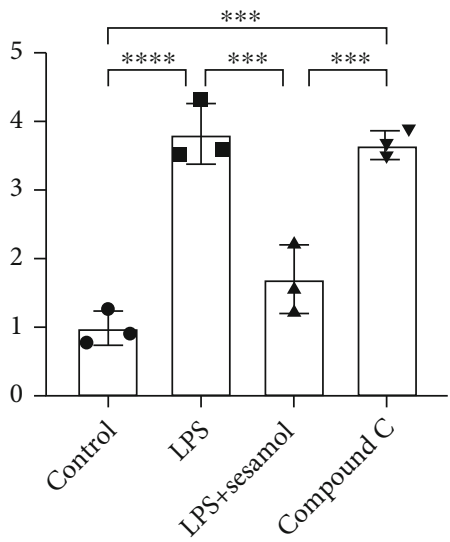

(h)

FIgURE 7: Sesamol regulates the AMPK/SIRT1/NF- $\kappa$ B pathway in BV2 cells suffering from LPS. (a) Western blot images showing that compound C reversed p-AMPK, AMPK, SIRT1, p-p65, and p65 protein expression regulated by sesamol in BV2 cells incubated with LPS. (b-d) Quantification of p-AMPK/AMPK ratio, SIRT1, and p-p65/p65 ratio data from (a) ( $n=3$ per group). (e) Representative images showing that compound C counteracted fluorescence intensity of SIRT1 (green) increased by sesamol (scale bar $=20 \mu \mathrm{m})$. (f) Representative images showing that sesamol decreased fluorescence intensity of nuclear p65 (red) while it was reversed by compound C (scale bar $=50 \mu \mathrm{m}) .(\mathrm{g}, \mathrm{h})$ Quantification of the fluorescence degree of SIRT1 and p65 in BV2 from (e) and (f), respectively $(n=3$ per group). Results are represented as the mean \pm SEM. ${ }^{\#} P<0.05,{ }^{\# \#} P<0.01,{ }^{\# \# \#} P<0.001$, and ${ }^{\# \# \#} P<0.0001$.

macrophage phenotype [49]. SIRT1, a member of the SIRT family, has antiaging and anti-inflammatory effects [50]. SIRT1 acts as a downstream target of the AMPK pathway, and it also inhibits the activation of NF- $\kappa \mathrm{B}$ [27]. Many evidences verify the inhibitory effect of the AMPK/SIRT1/NF$\kappa \mathrm{B}$ pathway on inflammation $[22,25,48]$. In the present study, compound $\mathrm{C}$, a specific inhibitor of AMPK, was used in BV2 cells suffering from LPS. Our results reveal that AMPK participates in inhibitory effect of sesamol on inflammation. Moreover, our data found that sesamol reduced protein levels of CD86 and iNOS which established M1 microglia, while it increased M2 phenotype microglial protein (CD206 and Arg-1) expressions in BV2 cells exposed to LPS, but these results could be reversed by compound C. Microglia are activated as M1-type microglia after SCI and then secrete proinflammatory factors, which aggravate neuronal apoptosis in an inflammatory environment and make tissue repair difficult [51]. Excitingly, sesamol can promote M2 phenotype polarization and decrease proinflammatory cytokine secretion, sequentially improve the inflammatory environment around the tissue, and consequently inhibit neuronal apoptosis and exert a neuroprotective role in mice following SCI. Finally, this study confirmed that sesamol could activate the AMPK/SIRT1 pathway and inhibit NF- $\kappa \mathrm{B}$ activation in vitro (Figure 7 ). Therefore, this study indicates that sesamol attenuates 
neuroinflammation and improves neuron survival partly through regulating the $\mathrm{AMPK} / \mathrm{SIRT} 1 / \mathrm{NF}-\kappa \mathrm{B}$ pathway in mice following SCI.

\section{Conclusion}

This study suggests that sesamol promotes polarization of M2 phenotype microglia, inhibits neuroinflammation, alleviates neuronal apoptosis, and improves locomotor functional recovery by activating the AMPK/SIRT1 pathway and inhibiting $\mathrm{NF}-\kappa \mathrm{B}$ activation in mice after SCI. Our results support the antineuroinflammatory effect of sesamol in SCI, and sesamol may be a potential therapeutic agent for the treatment of SCI.

\section{Data Availability}

All data supporting the conclusions of this manuscript are provided in the text and figures. Please contact the author for data requests.

\section{Conflicts of Interest}

The authors declare that the research was conducted in the absence of any commercial or financial relationships that could be construed as a potential conflict of interest.

\section{Authors' Contributions}

Xiaochu Feng, Xianghang Chen, Jian Xiao, and Keyong Ye conceived and designed the research. Xianghang Chen, Muhammad Zaeem, Lulu Chen, and Xiangxiang Chen performed the experiments. Xianghang Chen, Wanying Zhang, Liwan Song, and Ling Xie analyzed the data. Xianghang Chen and Ling Xie wrote the original manuscript. Joana Mubwandarikwa and Jian Xiao revised the manuscript. Xiaochu Feng and Xianghang Chen are the first two authors who contributed equally to this work.

\section{Acknowledgments}

This work was partially supported by a research grant from the National Natural Science Foundation of China (81972150, 81801233) and Zhejiang Provincial Natural Science Foundation (LR18H150001, LQ18H090008).

\section{References}

[1] C. Tohda and T. Kuboyama, "Current and future therapeutic strategies for functional repair of spinal cord injury," Pharmacology \& Therapeutics, vol. 132, pp. 57-71, 2011.

[2] K. L. Zhou, Y. F. Zhou, K. Wu et al., "Stimulation of autophagy promotes functional recovery in diabetic rats with spinal cord injury," Scientific Reports, vol. 5, no. 1, p. 17130, 2015.

[3] B. Yang, F. Zhang, F. Cheng et al., "Strategies and prospects of effective neural circuits reconstruction after spinal cord injury," Cell Death \& Disease, vol. 11, no. 6, p. 439, 2020.

[4] J. Dai, G. Y. Yu, H. L. Sun et al., "MicroRNA-210 promotes spinal cord injury recovery by inhibiting inflammation via the
JAK-STAT pathway," European Review for Medical and Pharmacological Sciences, vol. 22, no. 20, pp. 6609-6615, 2018.

[5] T. Gaojian, Q. Dingfei, L. Linwei et al., "Parthenolide promotes the repair of spinal cord injury by modulating M1/M2 polarization via the NF-kappaB and STAT $1 / 3$ signaling pathway," Cell death discovery, vol. 6, p. 97, 2020.

[6] Z. Zheng, Y. Wu, Z. Li et al., "Valproic acid affects neuronal fate and microglial function via enhancing autophagic flux in mice after traumatic brain injury," Journal of Neurochemistry, vol. 154, no. 3, pp. 284-300, 2020.

[7] H. Nakajima, K. Honjoh, S. Watanabe, A. Kubota, and A. Matsumine, "Distribution and polarization of microglia and macrophages at injured sites and the lumbar enlargement after spinal cord injury," Neuroscience Letters, vol. 737, article 135152, 2020.

[8] D. J. Hellenbrand, K. A. Reichl, B. J. Travis et al., "Sustained interleukin-10 delivery reduces inflammation and improves motor function after spinal cord injury," Journal of Neuroinflammation, vol. 16, no. 1, p. 93, 2019.

[9] Y. Ren and W. Young, "Managing inflammation after spinal cord injury through manipulation of macrophage function," Neural Plasticity, vol. 2013, Article ID 945034, 2013.

[10] L. Fan, K. Wang, Z. Shi, J. Die, C. Wang, and X. Dang, “Tetramethylpyrazine protects spinal cord and reduces inflammation in a rat model of spinal cord ischemia-reperfusion injury," Journal of Vascular Surgery, vol. 54, no. 1, pp. 192-200, 2011.

[11] J. L. Wang, C. H. Ren, J. Feng, C. H. Ou, and L. Liu, "Oleanolic acid inhibits mouse spinal cord injury through suppressing inflammation and apoptosis via the blockage of p38 and JNK MAPKs," Biomedicine \& Pharmacotherapy, vol. 123, article 109752, 2020.

[12] Y. Y. Wang, D. Shen, L. J. Zhao, N. Zeng, and T. H. Hu, "Sting is a critical regulator of spinal cord injury by regulating microglial inflammation via interacting with TBK1 in mice," Biochemical and Biophysical Research Communications, vol. 517, no. 4, pp. 741-748, 2019.

[13] K. Chopra, V. Tiwari, V. Arora, and A. Kuhad, "Sesamol suppresses neuro-inflammatory cascade in experimental model of diabetic neuropathy," The Journal of Pain, vol. 11, pp. 950957, 2010.

[14] B. Ren, T. Yuan, Z. Diao, C. Zhang, Z. Liu, and X. Liu, "Protective effects of sesamol on systemic oxidative stress-induced cognitive impairments via regulation of Nrf2/Keap1 pathway," Food \& Function, vol. 9, no. 11, pp. 5912-5924, 2018.

[15] A. F. Majdalawieh and Z. R. Mansour, "Sesamol, a major lignan in sesame seeds (Sesamum indicum): anti-cancer properties and mechanisms of action," European Journal of Pharmacology, vol. 855, pp. 75-89, 2019.

[16] N. D'Onofrio, L. Servillo, and M. L. Balestrieri, "SIRT1 and SIRT6 signaling pathways in cardiovascular disease protection," Antioxidants \& Redox Signaling, vol. 28, pp. 711-732, 2018.

[17] P. K. Bagul, N. Deepthi, R. Sultana, and S. K. Banerjee, "Resveratrol ameliorates cardiac oxidative stress in diabetes through deacetylation of NFkB-p65 and histone 3," The Journal of Nutritional Biochemistry, vol. 26, pp. 1298-1307, 2015.

[18] G. Hasko and P. Pacher, "Endothelial Nrf2 activation: a new target for resveratrol?,” American Journal of Physiology. Heart and Circulatory Physiology, vol. 299, pp. H10-H12, 2010.

[19] A. J. Donato, K. A. Magerko, B. R. Lawson, J. R. Durrant, L. A. Lesniewski, and D. R. Seals, "SIRT-1 and vascular endothelial 
dysfunction with ageing in mice and humans," The Journal of Physiology, vol. 589, no. 18, pp. 4545-4554, 2011.

[20] M. Li, N. Meng, X. Guo et al., "Dl-3-n-butylphthalide promotes remyelination and suppresses inflammation by regulating AMPK/SIRT1 and STAT3/NF- $\kappa$ B signaling in chronic cerebral hypoperfusion," Frontiers in Aging Neuroscience, vol. 12, p. 137, 2020.

[21] Z. Liu, M. Zhang, T. Zhou, Q. Shen, and X. Qin, "Exendin-4 promotes the vascular smooth muscle cell re-differentiation through AMPK/SIRT1/FOXO3a signaling pathways," Atherosclerosis, vol. 276, pp. 58-66, 2018.

[22] L. Chen and Z. Lan, "Polydatin attenuates potassium oxonateinduced hyperuricemia and kidney inflammation by inhibiting NF-kappaB/NLRP3 inflammasome activation via the AMPK/ SIRT1 pathway," Food \& Function, vol. 8, pp. 1785-1792, 2017.

[23] S. Bansod and C. Godugu, "Nimbolide ameliorates pancreatic inflammation and apoptosis by modulating NF-kappaB/ SIRT1 and apoptosis signaling in acute pancreatitis model," International Immunopharmacology, vol. 90, article 107246, 2021.

[24] Z. K. Wang, R. R. Chen, J. H. Li et al., "Puerarin protects against myocardial ischemia/reperfusion injury by inhibiting inflammation and the NLRP3 inflammasome: The role of the SIRT1/NF- $\kappa$ B pathway," International Immunopharmacology, vol. 89, article 107086, 2020.

[25] C. Wang, Y. Gao, Z. Zhang et al., "Safflower yellow alleviates osteoarthritis and prevents inflammation by inhibiting PGE2 release and regulating NF- $\kappa \mathrm{B} / \mathrm{SIRT} 1 / \mathrm{AMPK}$ signaling pathways," Phytomedicine, vol. 78, article ???, 2020.

[26] Y. C. Lu, T. Jayakumar, Y. F. Duann et al., "Chondroprotective role of sesamol by inhibiting MMPs expression via retaining NF- $\kappa$ B signaling in activated SW1353 cells," Journal of Agricultural and Food Chemistry, vol. 59, no. 9, pp. 4969-4978, 2011.

[27] X. L. Wu, C. J. Liou, Z. Y. Li, X. Y. Lai, L. W. Fang, and W. C. Huang, "Sesamol suppresses the inflammatory response by inhibiting NF- $\kappa \mathrm{B} / \mathrm{MAPK}$ activation and upregulating AMP kinase signaling in RAW 264.7 macrophages," Inflammation Research, vol. 64, no. 8, pp. 577-588, 2015.

[28] W. Ruankham, W. Suwanjang, P. Wongchitrat, V. Prachayasittikul, S. Prachayasittikul, and K. Phopin, "Sesamin and sesamol attenuate $\mathrm{H} 2 \mathrm{O} 2$-induced oxidative stress on human neuronal cells via the SIRT1-SIRT3-FOXO3a signaling pathway," Nutritional neuroscience., vol. 24, no. 2, pp. 90-101, 2021.

[29] A. Sekiguchi, H. Kanno, H. Ozawa, S. Yamaya, and E. Itoi, "Rapamycin promotes autophagy and reduces neural tissue damage and locomotor impairment after spinal cord injury in mice," Journal of Neurotrauma, vol. 29, no. 5, pp. 946956, 2012.

[30] D. M. Basso, L. C. Fisher, A. J. Anderson, L. B. Jakeman, D. M. Mctigue, and P. G. Popovich, "Basso mouse scale for locomotion detects differences in recovery after spinal cord injury in five common mouse strains," Journal of Neurotrauma, vol. 23, no. 5, pp. 635-659, 2006.

[31] Y. Li, J. Xiang, J. Zhang, J. Lin, Y. Wu, and X. Wang, "Inhibition of Brd4 by JQ1 promotes functional recovery from spinal cord injury by activating autophagy," Frontiers in Cellular Neuroscience, vol. 14, article 555591, 2020.

[32] T. Taetzsch, S. Levesque, C. McGraw et al., "Redox regulation of NF- $\kappa$ B p50 and M1 polarization in microglia," Glia, vol. 63, no. 3, pp. 423-440, 2015.
[33] Y. An, J. Li, Y. Liu, and M. Fan, "Neuroprotective effect of novel celecoxib derivatives against spinal cord injury via attenuation of COX-2, oxidative stress, apoptosis and inflammation," Bioorganic Chemistry, vol. 101, article 104044, 2020.

[34] B. Zhao, B. Xia, X. Li et al., "Sesamol supplementation attenuates DSS-induced colitis via mediating gut barrier integrity, inflammatory responses, and reshaping gut microbiome," Journal of Agricultural and Food Chemistry, vol. 68, no. 39, pp. 10697-10708, 2020.

[35] B. Ren, T. Yuan, X. Zhang et al., "Protective effects of sesamol on systemic inflammation and cognitive impairment in aging mice," Journal of Agricultural and Food Chemistry, vol. 68, no. 10, pp. 3099-3111, 2020.

[36] J. S. Wang, P. H. Tsai, K. F. Tseng, F. Y. Chen, W. C. Yang, and M. Y. Shen, "Sesamol Ameliorates Renal Injury-Mediated Atherosclerosis via Inhibition of Oxidative Stress/IKK $\alpha / \mathrm{p} 53$," Nutritional Neuroscience, vol. 10, no. 10, p. 1519, 2021.

[37] W. Han, Y. Li, J. Cheng et al., "Sitagliptin improves functional recovery via GLP-1R-induced anti-apoptosis and facilitation of axonal regeneration after spinal cord injury," Journal of Cellular and Molecular Medicine, vol. 24, no. 15, pp. 8687-8702, 2020.

[38] M. Srinivasan, B. Bayon, N. Chopra, and D. K. Lahiri, "Novel nuclear factor-kappaB targeting peptide suppresses betaamyloid induced inflammatory and apoptotic responses in neuronal cells," PLoS One, vol. 11, article e0160314, 2016.

[39] K. Karova, J. V. Wainwright, L. Machova-Urdzikova et al., "Transplantation of neural precursors generated from spinal progenitor cells reduces inflammation in spinal cord injury via NF- $\kappa$ B pathway inhibition," Journal of Neuroinflammation, vol. 16, no. 1, p. 12, 2019.

[40] D. J. Hines, R. M. Hines, S. J. Mulligan, and B. A. Macvicar, "Microglia processes block the spread of damage in the brain and require functional chloride channels," Glia, vol. 57, pp. 1610-1618, 2009.

[41] C. J. Heijnen and A. Kavelaars, "Neuro-immune, behavioral and molecular aspects of brain damage," Brain, Behavior, and Immunity, vol. 24, pp. 705-707, 2010.

[42] G. Khayrullina, S. Bermudez, and K. R. Byrnes, "Inhibition of NOX2 reduces locomotor impairment, inflammation, and oxidative stress after spinal cord injury," Journal of Neuroinflammation, vol. 12, p. 172, 2015.

[43] S. Xu, W. Zhu, M. Shao et al., "Ecto-5'-nucleotidase (CD73) attenuates inflammation after spinal cord injury by promoting macrophages/microglia M2 polarization in mice," Journal of Neuroinflammation, vol. 15, no. 1, p. 155, 2018.

[44] Y. Yang, Y. Qu, X. Lv et al., "Sesamol supplementation alleviates nonalcoholic steatohepatitis and atherosclerosis in high-fat, high carbohydrate and high-cholesterol diet-fed rats," Food \& Function, vol. 12, no. 19, pp. 9347-9359, 2021.

[45] K. Gourishetti, R. Keni, P. G. Nayak et al., "Sesamol-loaded PLGA nanosuspension for accelerating wound healing in diabetic foot ulcer in rats," International Journal of Nanomedicine, vol. 15, pp. 9265-9282, 2020.

[46] D. H. Lee, S. H. Chang, D. K. Yang, N. J. Song, U. J. Yun, and K. W. Park, "Sesamol increases Ucp1 expression in white adipose tissues and stimulates energy expenditure in high-fat diet-fed obese mice," Nutrients, vol. 12, no. 5, p. 1459, 2020.

[47] H. Qin, H. Xu, L. Yu, L. Yang, C. Lin, and J. Chen, "Sesamol intervention ameliorates obesity-associated metabolic 
disorders by regulating hepatic lipid metabolism in high-fat diet-induced obese mice," Food Nutrition Research, vol. 63, 2019.

[48] Y. Wang, Z. Yang, L. Yang et al., "Liuweidihuang pill alleviates inflammation of the testis via AMPK/SIRT1/NF-kappaB pathway in aging rats," Evidence-based Complementary and Alternative Medicine, vol. 2020, Article ID 2792738, 2020.

[49] X. Q. Xiong, Z. Geng, B. Zhou et al., "FNDC5 attenuates adipose tissue inflammation and insulin resistance via AMPKmediated macrophage polarization in obesity," Metabolism, vol. 83, pp. 31-41, 2018.

[50] X. Huang, Y. Shi, H. Chen et al., "Isoliquiritigenin prevents hyperglycemia-induced renal injuries by inhibiting inflammation and oxidative stress via SIRT1-dependent mechanism," Cell Death \& Disease, vol. 11, no. 12, p. 1040, 2020.

[51] X. Lu, F. Lu, J. Yu et al., "Gramine promotes functional recovery after spinal cord injury via ameliorating microglia activation," Journal of Cellular and Molecular Medicine, vol. 25, no. 16, pp. 7980-7992, 2021. 